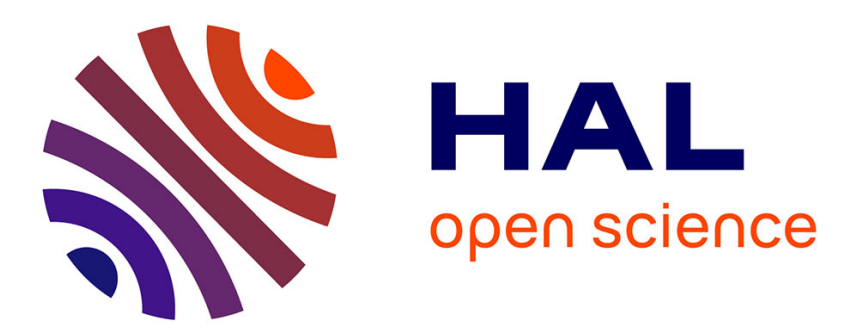

\title{
Analysis of collocated feedback controllers for four-bar planar mechanisms with joint clearances
}

\author{
Narendra Akhadkar, Vincent Acary, Bernard Brogliato
}

\section{To cite this version:}

Narendra Akhadkar, Vincent Acary, Bernard Brogliato. Analysis of collocated feedback controllers for four-bar planar mechanisms with joint clearances. Multibody System Dynamics, 2016, 38 (2), pp.101-136. 10.1007/s11044-016-9523-x . hal-01218531

\section{HAL Id: hal-01218531 \\ https://inria.hal.science/hal-01218531}

Submitted on 21 Oct 2015

HAL is a multi-disciplinary open access archive for the deposit and dissemination of scientific research documents, whether they are published or not. The documents may come from teaching and research institutions in France or abroad, or from public or private research centers.
L'archive ouverte pluridisciplinaire HAL, est destinée au dépôt et à la diffusion de documents scientifiques de niveau recherche, publiés ou non, émanant des établissements d'enseignement et de recherche français ou étrangers, des laboratoires publics ou privés. 


\title{
Analysis of collocated feedback controllers for four-bar planar mechanisms with joint clearances
}

\author{
Narendra Akhadkar* $\quad$ Vincent Acary $^{\dagger} \quad$ Bernard Brogliato ${ }^{\ddagger}$
}

June 22, 2015

Keywords: four-bar mechanism; clearance; dynamic backlash; unilateral constraints; Coulomb's friction; impacts; feedback control; passivity-based control; state feedback linearization; Moreau-Jean timestepping scheme;

\begin{abstract}
This article presents an analysis of two-dimensional four-bar mechanisms with joint clearance, when one joint is actuated by collocated open-loop or state feedback controllers (proportional-derivative, state feedback linearization, passivity-based control). The study is led with numerical simulations obtained with a projected Moreau-Jean's event-capturing algorithm. The contact/impact model uses kinematic coefficients of restitution, and Coulomb's friction. The focus is put on how much the performance deteriorates when clearances are added in the joints. It is shown that collocated feedback controllers behave in a very robust way.
\end{abstract}

\section{Introduction}

A four-bar mechanism is the simplest form of closed chain linkage. It is widely used in many industrial applications. A closed chain linkage may be used, for transmission or transformation of motion, to precisely reach the desired position or orientation. Usually the performance of a closed chain linkage is not as desired due to the manufacturing tolerances on links, clearance in the joints and the assembly tolerances. However the effects of clearance in the joints are different from link dimensional tolerances. The link dimensional tolerance leads to deviation in position and orientation which are predictable and repeatable. A joint clearance is a hard highly nonlinear disturbance inducing an increase of degrees of freedom, and it may lead to uncertainty in the output position and motion, which may deteriorate the performance of industrial applications [72].

These deviations between design and real behavior motivated many researchers in Mechanical Engineering [13, 14, 17, 21, 25, 28, 29, 36, 40, 58, 59, 64] to study the revolute joints with imperfections. Proper modeling of the joint clearances in multibody mechanical system is required to predict the behaviour of real systems. Different contact models and simulation tools are available [27]. In the

\footnotetext{
${ }^{*}$ Schneider Electric, 31 avenue Pierre Mendès France, 38320 Eybens, France. narendra.akhadkar@ schneider-electric.com.

${ }^{\dagger}$ INRIA Grenoble, Université Grenoble-Alpes, 655 avenue de l'Europe, Inovallée, 38334 Saint-Ismier, France. vincent.acary@inria.fr

${ }^{\ddagger}$ INRIA Grenoble, Université Grenoble-Alpes, 655 avenue de l’Europe, Inovallée, 38334 Saint-Ismier, France. bernard.brogliato@inria.fr
} 
experimental and numerical study of planar slider crank and four-bar mechanism with multiple revolute clearance joints [25, 29, 20, 18], the influence of clearance on performance of the system is demonstrated. The degradation of the system's performance is always in the form of vibration, noise, very high reaction forces at the joints, precision, and accuracy of the output. The dynamic response of the system due to the joint clearances is more complex and tends to be chaotic in some situations [19, 21, 51, 58, 61, 73, 57]. To control this chaotic behaviour, delayed feedback control [51], optimization of inertial effects [73], or redundant actuators that guarantee suitable preload for backlash avoidance in parallel manipulators [47], have been proposed.

In parallel with multibody modeling and numerical simulation, feedback controllers have been proposed with the purpose of increasing the motion accuracy of systems with clearances. This is called backlash compensation in the Systems and Control literature [49, 37]. Two major classes of models are used: dead-zone and hysteresis models, also called static backlash [66, 67, 10, 75], which are suitable for feedback control design but completely neglect the contact/impact dynamics, and dynamic backlash with compliant spring/dashpot models [48, 38]. Few studies use dynamic backlash with nonsmooth, setvalued models [32, 42]. Static and dynamic models of backlash yield quite different harmonic properties [12].

Most if not all of the multibody-oriented above studies, as well as some of the control-oriented ones, use the contact/impact phenomena in the clearances with compliant, linear or nonlinear spring/dashpot models (this is even sometimes stated as a basic modeling requirement [52]), and regularized Coulomb's friction [73, 41]. A major drawback of such an approach is that the numerical stabilization of contact forces and accelerations during the persistent contact phases, is not an easy task. Spurious oscillations may appear in the simulation of these contact modes (see e.g. [23, 35, 65, 50, 27, 73], [22, Figures $4.22,4.23]$ ). Moreover the regularization of Coulomb's law at zero tangential velocity (i.e. in the 2dimensional case, replacing the vertical segment of Coulomb's law characteristic by some finite-slope or sigmoid curve) has to be absolutely avoided since it cannot model properly the sticking modes which play a significant role in the contact dynamics. In addition, contrarily to what is sometimes stated [54], very efficient numerical methods exist for the simulation of set-valued characteristics, that we use in this work. Finally, the contact parameters estimation may be a hard task (especially if both normal and tangential models depend on several parameters, and impacts are considered), and stiff differential equations may appear due to very large contact equivalent stiffnesses. Therefore nonsmooth, set-valued models which use few parameters but retain the major contact dynamics features, may be preferred in many multibody multicontact applications.

Thümmel et al. [64], discussed the methodology for modeling mechanisms with clearance, friction and impact within the so-called nonsmooth contact dynamic method (NSCD) introduced by Moreau and Jean [43, 45, 46, 30, 31]: the interaction between bodies is modeled with unilateral constraints, complementarity conditions, kinematic or kinetic restitution coefficients, and set-valued frictional models (like Coulomb's law) [56, 26, 7]. Following Moreau [44], the dynamics of rigid multibody systems is formulated at the velocity-impulse level. The NSCD has proved to be a quite efficient numerical method, capable of handling complementarity conditions, as well as impacts and set-valued friction laws [3, 63]. Further studies using the nonsmooth contact dynamics methods may be found in [24, 36, 64]. Careful comparisons between numerical and experimental data are reported in [36, 64, 68, 69, 70, 71]: they show that the so-called time-stepping numerical schemes associated with set-valued force laws, possess very 
good forecast capabilities. This motivates us to use the NSCD method, with the enhanced scheme derived in [2] and available in the INRIA open-source library SICONOS [3]. It is noteworthy that all of the above analysis (as well as the one in this paper) deal with 2-dimensional joints. Recently the 3-dimensional case has been tackled in [74, 41]. In ushc a case cyindrical contact/impact models may be considered [55].

In this article three different examples of the four-bar mechanism (crank-rocker, crank-crank and rocker-rocker, see Figure 1) controlled with six different inputs are studied, mainly through numerical simulations. From a general point of view, joint clearances introduce nonsmooth, nonlinear perturbations and an increase of the system's degrees of freedom, which render the controlled system underactuated. Studying the robustness of (otherwise globally exponentially stable) controllers with respect to such hard disturbances, is a tough task, because analyzing the effects of impacts and friction on the closed-loop system's Lyapunov function derivative, is in general quite cumbersome. Our objective is not to derive new control strategies for backlash compensation, but to study both qualitatively and quantitatively how the addition of clearances modifies the controlled system's behaviour. Surprisingly enough, collocated feedback inputs possess remarkable robustness and drastically improve the system's performance compared with open-loop control torques.

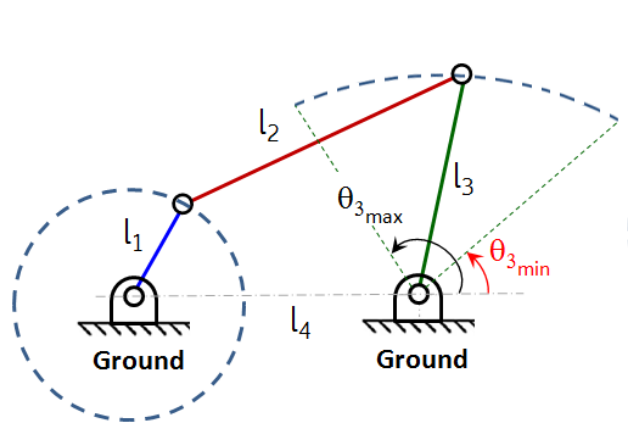

(a) Crank-rocker.

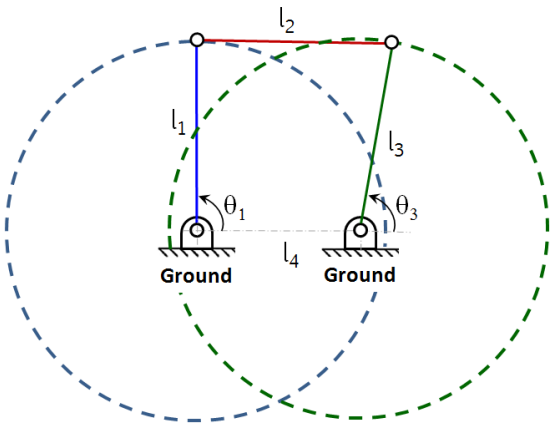

(b) Crank-crank.

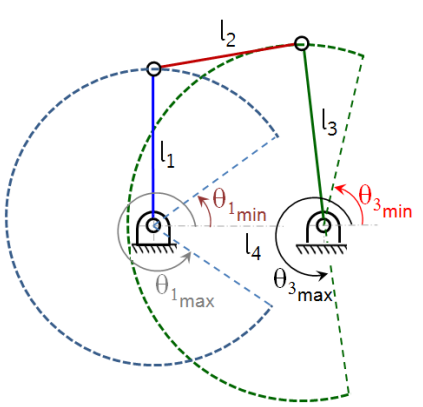

(c) Rocker-rocker.

Figure 1: Three types of four-bar mechanisms.

The article is organized follows: the dynamics are introduced in Section 2 , the local kinematics which allow to derive the gap functions in Section 2.1, the normal and tangential contact laws in Section 2.2 . the Lagrange dynamics in Section 2.3 and the numerical scheme in Section 2.4. Section 3 is dedicated to the analysis of the four-bar systems with time-dependent, open-loop control inputs. Four different feedback controllers are studied in Section 4, two Proportional-Derivative (PD) inputs in Section 4.1, a state feedback linearization in Section 4.2, and a passivity-based controller in Section 4.3. Conclusions end the article in Section 5. Details on the systems' dynamics are given in the Appendix.

\section{The Lagrange dynamics with unilateral constraints and Coulomb's fric- tion}

\subsection{Modeling of revolute joints with $2 \mathrm{D}$ clearance}

The local kinematics which allow to derive the unilateral constraints are treated in great details in [26, 56, 3]. Let us provide its formulation for a generic revolute joint with radial clearance $c$ as depicted 
on Figure 2. In an ideal revolute joint, it is assumed that the centers of two interconnected bodies (journal and bearing) are coincident. A revolute joint with clearance separates these two center points. It does

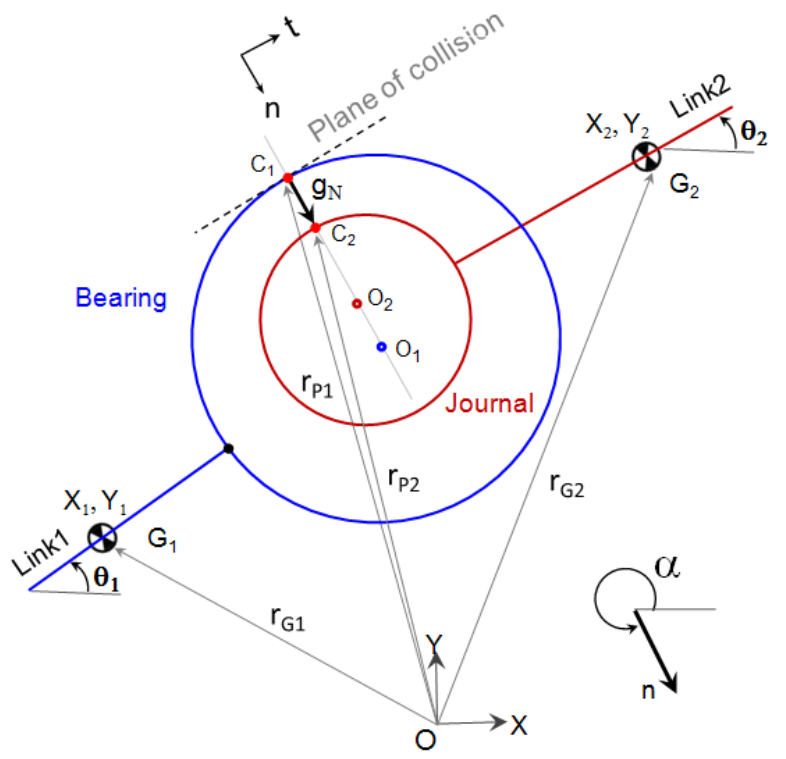

Figure 2: Planar revolute joint with clearance in a multibody system.

not constrain any degree of freedom in the mechanical system like the ideal revolute joint. However it imposes kinematic restrictions on the journal's motion. Thus an imperfect revolute joint introduces two degrees of freedom in the mechanical system. The radial clearance is defined as $c=r_{1}-r_{2}$, where $r_{1}$ is the radius of bearing and $r_{2}$ is the radius of journal $\left(r_{1}>r_{2}\right)$. On Figure 2, $O_{1}$ and $O_{2}$ indicate the bearing and journal centers, $C_{1}$ and $C_{2}$ represent the potential contact points on the bearing and journal respectively. The $(O, \mathbf{i}, \mathbf{j})$ coordinate frame represents the inertial coordinate system (with coordinates $X$ and $Y$ ). The vectors $r_{C_{1}}$ and $r_{C_{2}} \in \mathbb{R}^{2}$ are denoting the positions of contact points $C_{1}$ and $C_{2}$ in the inertial coordinate system. The centers of mass of bodies 1 and 2 are $G_{1}$ and $G_{2}$, with coordinates $\left(X_{1}, Y_{1}\right)$ and $\left(X_{2}, Y_{2}\right)$ respectively. The bodies orientations are the angles $\theta_{1}$ and $\theta_{2}$. The vectors $r_{G_{1}}$ and $r_{G_{2}} \in \mathbb{R}^{2}$ denote the positions of the bearing and journal's centers of mass, while $r_{O_{1}}$ and $r_{O_{2}} \in \mathbb{R}^{2}$ denote the positions of the centers of bearing and journal, both in the inertial coordinate system. The normal and tangential vectors to the plane of collision between the bearing and the journal are defined by $(\mathbf{n}, \mathbf{t}) \in \mathbb{R}^{2}$. Note that the unit vector $\mathbf{n}$ has the same direction as the line of the centers of the journal and the bearing. The orientation of $\mathbf{n}$ is chosen such that it always acts inward from journal center to bearing center. The signed distance (or gap function) is calculated as:

$$
g_{N}=C_{1} C_{2} \mathbf{n}=c-O_{2} O_{1} \mathbf{n}
$$


The magnitude of eccentricity (clearance) vector $O_{2} O_{1}$ is denoted by $\left\|O_{2} O_{1}\right\|$ and its orientation is given by $\alpha$. The unit normal vector $\mathbf{n}$ is given as $\mathbf{n}=\frac{\mathrm{O}_{2} \mathrm{O}_{1}}{\left\|\mathrm{O}_{2} \mathrm{O}_{1}\right\|}$, with:

$$
\begin{aligned}
& O_{2} O_{1}=\left(X_{1}+\frac{l_{1}}{2} \cos \theta_{1}-X_{2}+\frac{l_{2}}{2} \cos \theta_{2}\right) \mathbf{i}+\left(Y_{1}+\frac{l_{1}}{2} \sin \theta_{1}-Y_{2}+\frac{l_{2}}{2} \sin \theta_{2}\right) \mathbf{j} \\
& \mathbf{n}=\cos \alpha \mathbf{i}+\sin \alpha \mathbf{j}, \quad \mathbf{t}=-\sin \alpha \mathbf{i}+\cos \alpha \mathbf{j} \\
& \cos \alpha=\left(\frac{X_{1}+\frac{l_{1}}{2} \cos \theta_{1}-X_{2}+\frac{l_{2}}{2} \cos \theta_{2}}{\left\|O_{2} O_{1}\right\|}\right), \quad \sin \alpha=\left(\frac{Y_{1}+\frac{l_{1}}{2} \sin \theta_{1}-Y_{2}+\frac{l_{2}}{2} \sin \theta_{2}}{\left\|O_{2} O_{1}\right\|}\right)
\end{aligned}
$$

If we denote the generalized coordinates of each body as $q_{i}=\left(X_{i}, Y_{i}, \theta_{i}\right)^{T}, i=1,2$, then we obtain that $g_{N}=g_{N}\left(q_{1}, q_{2}\right)$. We also have $r_{C_{1}}=r_{G_{1}}+G_{1} C_{1}=r_{G_{1}}+G_{1} O_{1}+O_{1} C_{1}$ and $r_{C_{2}}=r_{G_{2}}+G_{2} C_{2}=$ $r_{G_{2}}+G_{2} O_{2}+O_{2} C_{2}$. Differentiating these expressions with respect to time yields :

$$
\left\{\begin{array}{l}
V_{C_{1}}=\frac{d}{d t} r_{G_{1}}+\frac{d}{d t}\left(G_{1} C_{1}\right)=\frac{d}{d t} r_{G_{1}}+\frac{d}{d t}\left(G_{1} O_{1}\right)+\frac{d}{d t}\left(O_{1} C_{1}\right) \\
V_{C_{2}}=e \frac{d}{d t} r_{G_{2}}+\frac{d}{d t}\left(G_{2} C_{2}\right)=\frac{d}{d t} r_{G_{2}}+\frac{d}{d t}\left(G_{2} O_{2}\right)+\frac{d}{d t}\left(O_{2} C_{2}\right)
\end{array}\right.
$$

which leads to:

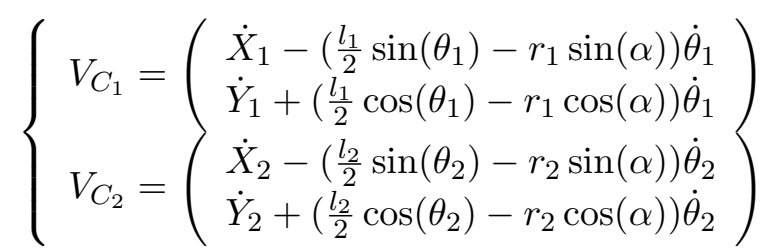

where $V_{C_{i}},(i=1,2) \in \mathbb{R}^{2}$ are the absolute velocities of the contact points. Consequently, the contact points relative velocity is expressed in the local frame as:

$$
U=\left(\begin{array}{c}
U_{N} \\
U_{T}
\end{array}\right)=\left(\begin{array}{c}
\left(V_{C_{2}}-V_{C_{1}}\right)^{T} \mathbf{n} \\
\left(V_{C_{2}}-V_{C_{1}}\right)^{T} \mathbf{t}
\end{array}\right)
$$

From (6) and (7) the normal and tangential components of the relative velocity can be calculated:

$$
\left(\begin{array}{c}
U_{N} \\
U_{T}
\end{array}\right)=\left(\begin{array}{cccccc}
\cos \alpha & \sin \alpha & \frac{l_{1}}{2} \sin A & -\cos \alpha & -\sin \alpha & \frac{l_{2}}{2} \sin B \\
-\sin \alpha & \cos \alpha & -\frac{l_{1}}{2} \cos A+r_{1} & \sin \alpha & -\cos \alpha & -\frac{l_{2}}{2} \cos B-r_{2}
\end{array}\right)\left(\begin{array}{c}
\dot{q}_{1} \\
\dot{q}_{2}
\end{array}\right)
$$

where $A=\left(\theta_{1}-\alpha\right), B=\left(\theta_{2}-\alpha\right)$.

\subsection{Normal and tangential contact laws}

The contact force is denoted $R=\left(R_{N}, R_{T}\right)^{T} \in \mathbb{R}^{2}$ in the local frame $(\mathbf{n}, \mathbf{t})$. Due to the impenetrability assumption one has $g_{N}(q) \geqslant 0$. We also neglect adhesive effects so that $R_{N} \geqslant 0$. If $R_{N}>0$ then we impose $g_{N}(q)=0$, and when $g_{N}(q)>0$, the normal contact force must vanish, i.e. $R_{N}=0$ (no magnetic or distance forces) [1, 3, 7]. These conditions yield a complementarity condition denoted compactly as:

$$
0 \leqslant g_{N}(q) \perp R_{N} \geqslant 0
$$

The normal contact law at the velocity level is expressed as :

$$
0 \leqslant U_{N}^{+}+e_{r} U_{N}^{-} \quad \perp \quad R_{N} \geqslant 0, \quad \text { if } g_{N}(q)=0
$$


where $U_{N}^{+}=\nabla g_{N}(q) \dot{q}^{+}$is the relative velocity after the collision, $U_{N}^{-}=\nabla g_{N}(q) \dot{q}^{-}$is the relative velocity before the collision, and $e_{r} \in[0,1]$ is the restitution coefficien(1 1 . The tangential contact law is based on Coulomb's friction law and it is defined locally at each contact point $\left(C_{1}=C_{2}\right)$. In the 2D case Coulomb's friction law is as follows:

$$
-R_{T} \in \mu\left|R_{N}\right| \operatorname{sgn}\left(U_{T}\right)
$$

where $\mu \geqslant 0$ is the coefficient of friction and $\operatorname{sgn}(\cdot)$ is the set-valued signum function with $\operatorname{sgn}(0)=$ $[-1,1]$. It is noteworthy that the basic Coulomb's law can be easily enhanced with static and dynamic friction coefficients, varying friction coefficient (with Stribeck effects), or micro-displacements during sticking modes, while staying in a set-valued context that is suitable for a proper time-discretization including sticking modes [3, §3.9].

\subsection{Lagrangian formulation with bilateral and unilateral constraints}

Let us consider a Lagrangian mechanical system with generalized coordinate vector $q \in \mathbb{R}^{n}$, and subjected to $m$ constraints, with $m_{b}$ holonomic bilateral constraints $g_{N}^{\alpha}=0, \alpha \in \mathcal{E}$, and $m_{u}$ unilateral constraints $g_{N}^{\alpha} \geqslant 0, \alpha \in \mathcal{I}, m=m_{b}+m_{u}=|\mathcal{E}|+|\mathcal{I}|$, and with 2D Coulomb friction. The Lagrangian formalism of such a system is as follows [3, 56],

$$
\left\{\begin{array}{l}
\dot{q}(t)=v(t), \\
M(q(t)) \dot{v}(t)+F(t, q(t), v(t))=G_{N}^{\top}(q(t)) R_{N}+G_{T}^{\top}(q(t)) R_{T}, \\
g_{N}^{\alpha}(q(t))=0, \quad \alpha \in \mathcal{E}, \\
g_{N}^{\alpha}(q(t)) \geqslant 0, \quad R_{N}^{\alpha} \geqslant 0, \quad R_{N}^{\alpha} g_{N}^{\alpha}(q(t))=0, \\
U_{N}^{\alpha}\left(t^{+}\right)=-e_{r}^{\alpha} U_{N}^{\alpha}\left(t^{-}\right), \text {if } g_{N}^{\alpha}(q(t))=0 \text { and } U_{N}^{\alpha}\left(t^{-}\right) \leqslant 0, \\
-R_{T}^{\alpha} \in \mu^{\alpha} R_{N}^{\alpha} \operatorname{sgn}\left(U_{T}^{\alpha}\right), \text { if } g_{N}^{\alpha}(q(t))=0 .
\end{array}\right\} \in \mathcal{I}
$$

where $v(t)$ is the vector of generalized velocities, $M(q) \in \mathbb{R}^{n \times n}$ is the mass matrix, $F(t, q, v)=$ $C(q, v) v-\mathbf{g}(q)-B \tau(t, q, v) \in \mathbb{R}^{n}$ is the vector of generalized forces, $C(q, v) \in \mathbb{R}^{n}$ is the vector of Coriolis and gyroscopic forces, $\mathbf{g}(q)$ contains forces which derive from a potential, $B \in \mathbb{R}^{n}$ is the input matrix, $\tau(t, q, v)$ is the scalar control torque applied at joint $J_{1}$ (see Figure 3 below), $G_{N}(q) \in \mathbb{R}^{m \times n}$ and $G_{T}(q) \in \mathbb{R}^{m \times n}$ are the linear maps of local normal and tangent frames at the contact points (i.e. $U_{T}=G_{T}(q) \dot{q}$ and $U_{N}=G_{N}(q) \dot{q}$, see (8)).

In the sequel only unilateral constraints will be considered, since bilateral constraints are eliminated by coordinate reduction. Details on the dynamics of the four-bar systems are provided in Appendices A B and C.

Remark 1. (i) The mathematical well-posedness of the Lagrange dynamics in (12) has been shown in the frictionless case in [15, 16. 53] 5]; in the case with friction see [6. 62]. (ii) When there is no clearance, $n=1$ and the system is fully actuated. When one (resp. two) clearance is present, $n=3$ (resp. $n=5$ ) and the system becomes underactuated. (iii) Various contact/impact models are compared in [22]. It is not obvious to determine which model is the best. The approach chosen in this article seems to be a

\footnotetext{
${ }^{1}$ When friction is present during impacts, there is in general no reason that $e_{r}$ should be upper bounded by 1 , see [7] Chapter 4]. Moreover inertial couplings may introduce kinetic energy increase for nearly elastic impacts. Finally dynamical singularities like Painlevé paradoxes may occur during sliding motions [7, Chapter 5]. We have not noticed such issues in the particular cases treated below, with small friction coefficients.
} 
suitable compromise for many physical effects occurring in joints with clearance, and which are quite difficult to encapsulate in a single contact/impact model with a reliable numerical method (dissipation at impacts, friction, conforming/non conforming contacts). As alluded to above it may be enhanced while staying in the same overall rigid body framework.

\subsection{The numerical integration method}

The numerical time-integration scheme used in this article is an event-capturing time-stepping method mainly based on the Moreau-Jean time-stepping scheme [43, 45, 46, 30, 31]. As we said in the introduction, the method uses a formulation of the dynamics at the velocity/impulse level, that enables a very robust numerical time-integration of systems with a lot of impact events. Contrary to event-driven schemes, the events are not accurately located in time but integrated within the time-step. Although it leads to robust schemes, the treatment of the constraints and the impact law at the velocity level yields drift at the position level. When we study multibody systems with clearances in joints with unilateral contact, we need to keep the drift of the constraints as small as possible with respect to the characteristic lengths of the clearances.

This is the reason why we use a scheme that satisfies constraints both at the velocity and position levels. It is an extension of the Moreau-Jean scheme together with the Gear-Gupta-Leimkuhler (GGL) method to systems with unilateral constraints and impacts [2]. Applying directly the GGL approach to unilateral constraint may yield to spurious oscillations at contact that depend on the activation procedure of the constraints at the velocity level. In [2], this issue is fixed by consistently activating the constraints within the time-step in an iterative way. Especially, we want to avoid the projection onto a constraint if the associated constraint at the velocity level is not activated. The so-called "combined scheme" is based on the iterations denoted by $\nu$ of the following two steps :

1. The projection step is based on the solution of the following system

$$
\left\{\begin{array}{l}
M\left(q_{k+\theta}\right)\left(v_{k+1}-v_{k}\right)-h F_{k+\theta}=G\left(q_{k+1}\right) P_{k+1}, \\
q_{k+1}=q_{k}+h v_{k+\theta}+G\left(q_{k+1}\right) \gamma_{k+1}, \\
U_{k+1}=G^{\top}\left(q_{k+1}\right) v_{k+1}, \\
g_{k+1}=g\left(q_{k+1}\right), \\
\text { for all } \alpha \in \mathcal{I}^{\nu}\left\{\begin{array}{l}
0 \leqslant U_{N, k+1}^{\alpha}+e U_{N, k}^{\alpha} \perp P_{N, k+1}^{\alpha} \geqslant 0, \\
-P_{T, k+1} \in \mu^{\alpha} P_{N, k+1}^{\alpha} \operatorname{sgn}\left(U_{T, k+1}^{\alpha}\right) \\
g_{k+1}^{\alpha}=0, \gamma_{k+1}^{\alpha}, \text { if } P_{N, k+1}^{\alpha}>0, \\
0 \leqslant g_{k+1}^{\alpha} \perp \gamma_{k+1}^{\alpha} \geqslant 0 \text { otherwise. }
\end{array}\right.
\end{array}\right.
$$

for a given index set $\mathcal{I}^{\nu}$ of active constraints. The time-step is denoted by $h$ and the notation $x_{k+\theta}=(1-\theta) x_{k}+\theta x_{k+1}$ is used for $\theta \in[0,1]$. Compared to the Moreau-Jean scheme, the multiplier $\gamma_{k+1}$ is added to improve the constraint drift. Note that $P_{k+1}$ is an impulse which remains always bounded when an impact occurs.

2. The activation step computes the index set $\mathcal{I}^{\nu}$ of active constraints by checking for a given value of $g_{k+1}$ if the constraint is satisfied or not. Starting form $\mathcal{I}^{0}=\emptyset$, at each iteration $\nu$, the activation 
performs the following operation

$$
\mathcal{I}^{\nu+1}=\mathcal{I}^{\nu} \cup\left\{\alpha \in \mathcal{I} \mid g_{k+1}^{\alpha} \leqslant 0\right\}
$$

The iterates $\left(q_{k+1}, v_{k+1}\right)$ of the solution depend on the iteration number $\nu$. In order to avoid useless complexity in the notation, we skip the superscript $\nu$ when there is no ambiguity. The steps 1 and 2 are iterated until the index set $\mathcal{I}^{\nu}$ is constant. The algorithm can be extended straightforwardly to the frictional case.

The contact events are not detected with high precision in such event-capturing methods, and the number of calculated impacts depends on $h$. In the next section the choice $h=10^{-5}$ s is chosen. Computations reported in [3, Table 14.2] show that this is a reasonable time step and smaller $h$ is not necessary, because the collisions which are not detected have negligible influence on the system's dynamics (in particular on the kinetic energy loss). The simulations in this article have been led with the code implemented in the INRIA open-source software SICONOS ${ }^{2}$

Remark 2. Two major classes of numerical methods exist: event-driven and event-capturing (or timestepping) schemes. They both possess advantages and drawbacks. In case of systems which undergo a large number of events (like stick/slip transitions and impacts), event-capturing methods are preferable despite their low-order [3] 63], because event-driven strategies rapidly become cumbersome to implement and too time-consuming. Moreover event-capturing methods have been proved to converge.

\subsection{Analysis methodology}

Let us consider a four-bar mechanism (see Figure 3 (a)-(b)) with bodies mass $m_{i}$, length $l_{i}$, inertia $I_{i}$, $1 \leqslant i \leqslant 3$. An imperfect joint is defined by a unilateral constraint $g_{j}=\left(c_{j}-O_{j} O_{j-1} \mathbf{n}\right) \geqslant 0, j=2$ or 3 , where $c_{j}$ is the radial clearance at the imperfect joint. The four-bar mechanism with clearance in one revolute joint is described by three generalized coordinates $q=\left[\theta_{1}, \theta_{2}, \theta_{3}\right]^{T}$, and with clearance in two revolute joints it is described by five generalized coordinates $q=\left[\theta_{1}, \theta_{2}, \theta_{3}, X_{2}, Y_{2}\right]^{T}$. The four-bar mechanism is actuated at the joint $1\left(J_{1}\right)$. We consider joints $J_{1}$ and $J_{4}$ to be perfect revolute joints while the joints $J_{2}$ and $J_{3}$ may be imperfect with radial clearance $c_{2}$ and $c_{3}$. The influence of different clearance sizes $c_{2}$ and $c_{3}$, coefficient of restitution $\left(e_{r}\right)$ and coefficient of friction $(\mu)$ on the mechanism performance is studied. Results are compared with the cases without clearance and without friction. The presence of clearance in the revolute joint can lead to variation in the initial conditions and this variation depends on the value of the radial clearance. To this aim, in the first step we study the influence of the initial conditions on the system's long term behaviour with perfect revolute joints. Let $\|\cdot\|_{\infty}$ be defined as ${ }^{3}\|X\|_{\infty}=\max _{t \in[1,10]}|X(t)|$. The percentage relative error in the angular position $\theta_{1}(0)$ is given as:

$$
e_{0}=\frac{\left\|\theta_{1}^{i 1}(t)-\theta_{1}^{i d l}(t)\right\|_{\infty}}{\left\|\theta_{1}^{i d l}(t)\right\|_{\infty}} \times 100
$$

where $\theta_{1}^{i d l}(t)$ is the angular position of links with the reference initial condition, and $\theta_{1}^{i 1}(t)$ is the angular position of links with different initial conditions. We plot the isolines of the percentage relative error $e_{0}$ with $\theta_{1}(0)$ and $\dot{\theta}_{1}(0)$. In the second step, we analyze through numerical simulations how much the

\footnotetext{
${ }^{2}$ http://siconos.gforge.inria.fr/

${ }^{3}$ The first initial period $[0,1] \mathrm{s}$ is not included in the infinity norm in order to eliminate the transient period, and concentrate on the steady-state behaviour of trajectories only.
} 


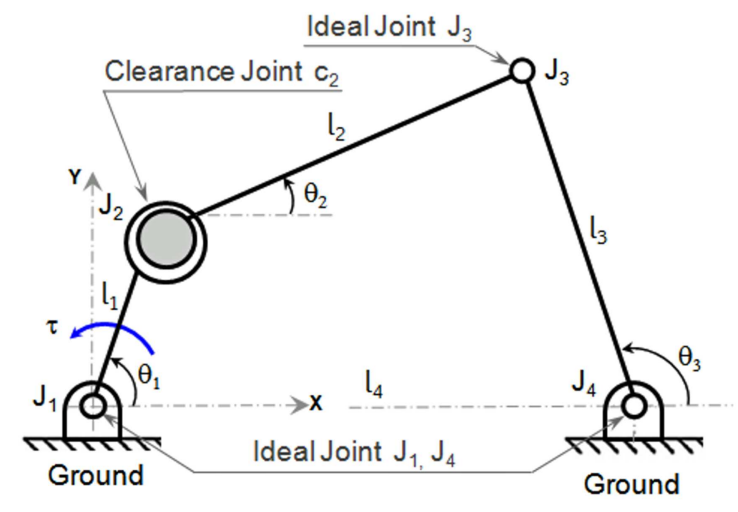

(a) Clearance in joint $J_{2}$.

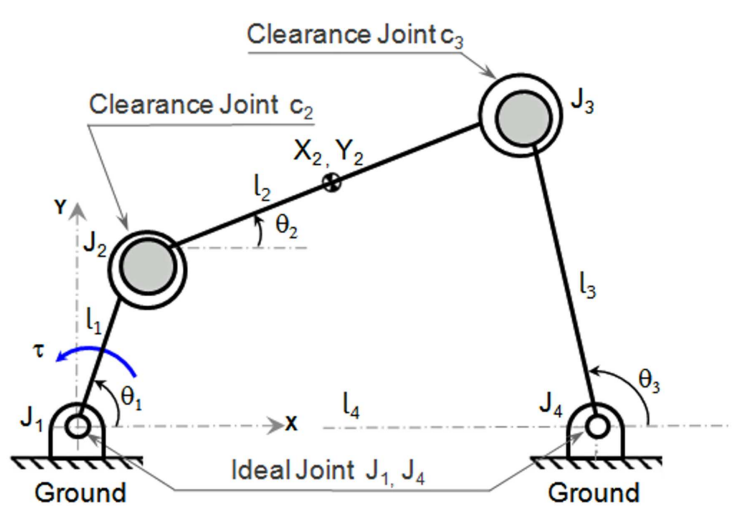

(b) Clearance in joint $J_{2}$ and $J_{3}$.

Figure 3: Four-bar mechanism with clearance in revolute joints.

presence of clearances deteriorates the system's dynamical behaviour. The percentage relative error in the angular positions $\theta_{1}$ and $\theta_{3}$ is given as:

$$
e=\max _{p \in\{1,3\}} \frac{\left\|\theta_{p}^{c l}(t)-\theta_{p}^{i d}(t)\right\|_{\infty}}{\left\|\theta_{p}^{i d}(t)\right\|_{\infty}} \times 100
$$

where $\theta_{p}^{i d}(t)$ is the angular position of links without joint clearance and $\theta_{p}^{c l}(t)$ is the angular position of links with joint clearance. The contour plot with different levels of isolines represents the variation of error in the angular position. In the second step, the initial conditions remain constant and only radial clearances $\left(c_{2}\right.$ and $\left.c_{3}\right)$ are varied for different values of coefficients of restitution $e_{r}$ and of friction $\mu$. For all contour plots, simulations are carried out for every $0.5 \mathrm{~mm}$ increment in joint clearance and for every 0.1 increment in coefficient of restitution. Therefore the error $e$ allows us to analyze the loss of performance of a controller when clearances are added, and is different from the usual tracking error that is widely used in the Control literature. It measures the proximity between the cases with and without mechanical play.

\section{Open-loop control}

In this section two open-loop ${ }^{4}$ inputs $\tau$ are considered: a constant torque $\tau_{1}=6.0 \mathrm{~N} \mathrm{~m}$ and a sinusoidal torque $\tau_{2}=9.0 \sin (0.75 \pi \mathrm{t}) \mathrm{N} \mathrm{m}$, applied at the joint $J_{1}$ in counter-clockwise direction. Since our main goal is comparison of feedback controllers, and since the results we obtained for the three types of four-bar mechanisms were quite similar, only the crank-rocker case is presented. Let us consider a crank-rocker mechanism as on Figure 1(a), where the input link $l_{1}$ rotates fully $\left(360^{\circ}\right)$ and the output link $l_{3}$ oscillates through angles $\theta_{3_{\min }}$ and $\theta_{3_{\max }}$. Geometric and inertial properties of the crank-rocker four-bar mechanism are given in Table 1 . The initial conditions are $\theta_{1}(0)=1.571 \mathrm{rad}, \theta_{2}(0)=0.3533 \mathrm{rad}$, $\theta_{3}(0)=1.2649 \mathrm{rad}, \dot{\theta}_{1}(0)=\dot{\theta}_{2}(0)=\dot{\theta}_{3}(0)=0.0 \mathrm{rad} / \mathrm{s}$. The coordinates of the center of gravity of link 2 are $X_{2}=1.8764 \mathrm{~m}, Y_{2}=1.6919 \mathrm{~m}$. Parameters used for the dynamic simulation are given in Table 2. The deviation in the system's performance is studied with the percentage relative error in angular position $e_{0}$ in 15 to find out the sensitivity to the initial conditions. The results are depicted on

\footnotetext{
${ }^{4}$ The name open-loop control means that the torque $\tau$ is a function of time only, with no position or velocity feedback.
} 
Table 1: Geometric and inertial properties of the crank-rocker four-bar mechanism.

\begin{tabular}{cccc}
\hline \hline Body Nr. & Length $[\mathrm{m}]$ & Mass $[\mathrm{kg}]$ & Inertia $\left[\mathrm{kg} \mathrm{m}^{2}\right]$ \\
\hline 1 & 1.0 & 1.0 & $8.33 \cdot 10^{-2}$ \\
2 & 4.0 & 1.0 & 1.33 \\
3 & 2.5 & 1.0 & $5.21 \cdot 10^{-1}$ \\
4 & 3.0 & & \\
\hline
\end{tabular}

Table 2: Parameters used in simulations.

\begin{tabular}{llll}
\hline \hline Nominal bearing radius $r_{2}$ & $0.06 \mathrm{~m}$ & Coefficient of restitution $e_{r}$ & {$[0,0.9]$} \\
Radial Clearance $c_{2}\left(\right.$ or $\left.c_{3}\right)$ & {$\left[0.0,5 \cdot 10^{-3}\right] \mathrm{m}$} & Time step $h$ & $1 \cdot 10^{-5} \mathrm{~s}$ \\
Coefficient of friction $\mu$ & $\{0.0,0.1\}$ & Total time of simulation $T$ & $10 \mathrm{~s}$ \\
\hline
\end{tabular}

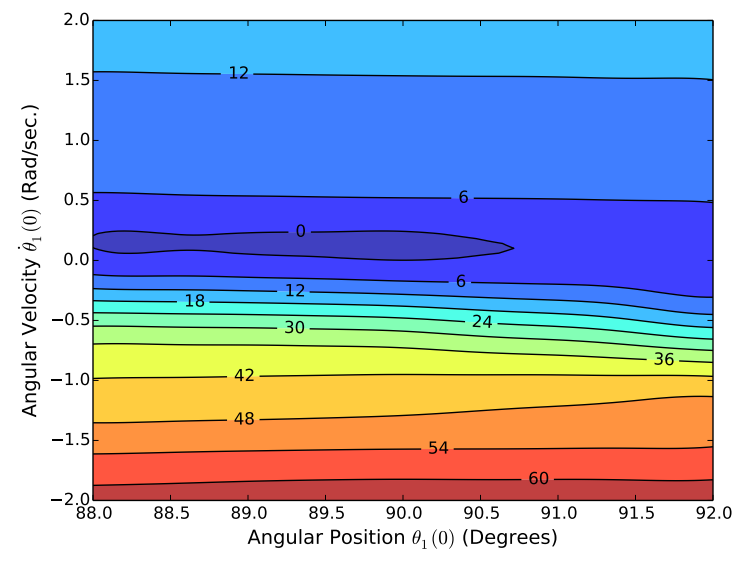

(a) Ideal case, $\tau=\tau_{1}$.

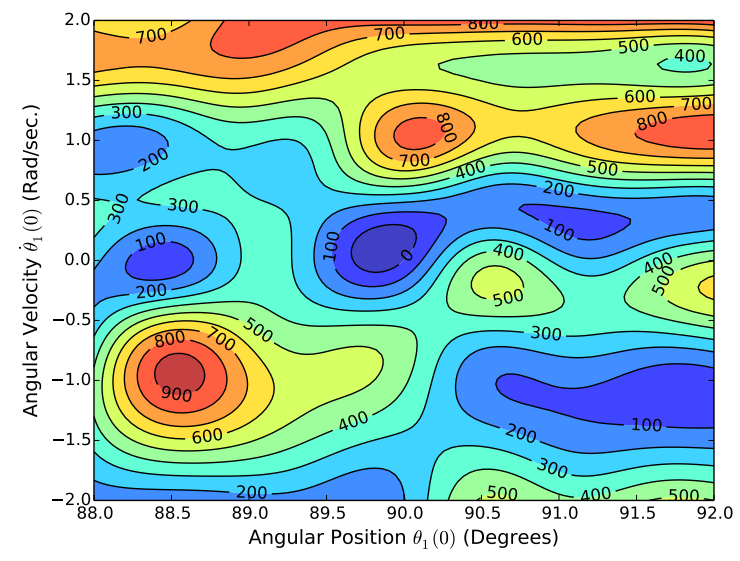

(b) Ideal case, $\tau=\tau_{2}$.

Figure 4: Crank-rocker with ideal joints: contour plot of $e_{0}$ with $\theta_{1}(0)$ and $\dot{\theta}_{1}(0)$.

Figure 4. The major conclusion is that the system's sensitivity w.r.t. initial conditions changes drastically when the constant torque is replaced by a sinusoidal one: Figure 4 (a) shows an ordered behaviour with horizontal stripes (zero gradient of $\left.e_{0}\left(\theta_{1}(0)\right)\right)$ and small gradient of $e_{0}\left(\dot{\theta}_{1}(0)\right)$, while Figure 4 (b) shows a disordered behaviour with a high gradient of $e_{0}\left(\theta_{1}(q), \dot{\theta}_{1}(0)\right)$ between the isolines, indicating high sensitivity.

Let us now analyze the case with one clearance in joint $J_{2}$. The numerical simulations are depicted on Figures 5, 6 and 7. On Figure 6, the trajectories $\theta_{1}(t)$ for various clearances, as well as the variables $g_{N}(q(t))$ and $\dot{g}_{T}(q(t))$ are depicted. The normal contact force $R_{N}(t)$ is also given for the case without friction. Finally the isolines of the percentage relative error $e$ as given in 16 are plotted and depicted on Figure 5. The results have been obtained, as indicated in Table 2, for the range of values of restitution coefficient $e_{r} \in[0.0,0.9]$. Only one set of simulations for $e_{r}=0.0$ is shown on Figure 6, because changing the restitution coefficient did not change the results significantly in agreement with the results on Figure 


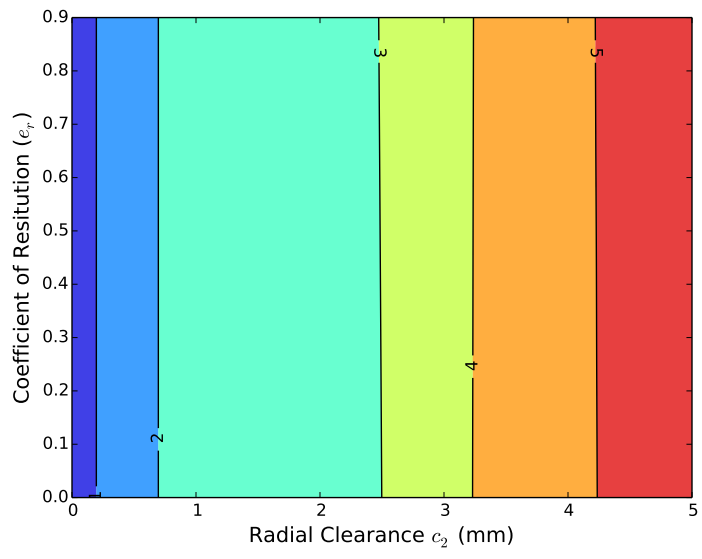

(a) Without friction, $\mu=0.0$.

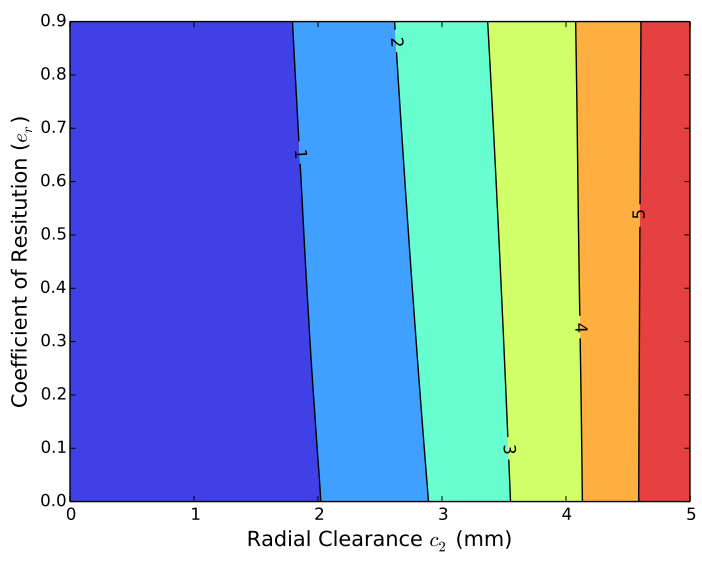

(b) With friction, $\mu=0.1$.

Figure 5: Crank-rocker with clearance in $J_{2}$ : contour plot of $e$ with $c_{2}$ and $e_{r}, \tau_{1}$.

5. The major conclusions are: (i) For the input torque $\tau_{1}$, the impacts and so the restitution coefficient $e_{r}$ play a negligible role for fixed clearance (vertical stripes on Figure 5). This may be attributed to too small values of the pre-impact velocities, and to a small number of collisions (see the plots of $g_{N}(q)$ on Figures 6 (a) (b) (c)). Figures 7 also illustrate that the rebound/contact inside the bearing is confined to small collisions mainly on one side of the bearing, almost independently of $e_{r}$. (ii) The maximum values taken by $g_{N}(q)$ after impacts are most of the time really smaller than the clearance $(5 \mathrm{~mm}$ on Figures 6 (a) (b) (c)), in agreement with Figure 7 (iii) The combined projection scheme in Algorithm ?? allows to simulate persistent contact phases without spurious oscillations, and very small drift. This is particularly visible on Figures 6(a)-(c) (see $g_{N}(q(t))$ between the peaks). (iv) For the torque $\tau_{2}$, the system's trajectories (see $\theta_{1}$ on Figure 6(c)-(d)) start deviating from a specific configuration marked as $P_{1}$ on the plot and after this point the system starts behaving randomly. This is common behaviour observed in systems with unilateral constraints and impacts (see e.g. [76, Figures 11, 12], see [39, 11] in the broader context of bifurcation and chaos analysis). (v) Surprisingly enough, the number of impacts with the sinusoidal input torque $\tau_{2}$ is smaller than with $\tau_{1}$ (see $g_{N}(q)$ on Figures 6(a) and (c)). (vi) As seen on Figure6(b), the system undergoes few stick/slip transitions in the joint $J_{2}\left(\dot{g}_{T}(q(t))\right.$ is almost always positive) but many variations of the tangential velocity at contact. (vii) For the driving torque $\tau_{1}$, the presence of small friction does not modify much the dynamical behaviour (see Figure 5 and $g_{N}(q)$ on Figures 6(a)-(b)).

Let us now consider now the crank-rocker mechanism with clearance in joints $J_{2}$ and $J_{3}$ (see Figure $3(\mathrm{~b})$ ). The isolines of the percentage relative error as given in $(16)$ are plotted for the radial clearance $c_{2}$ and $c_{3}$. The results for the input torques $\tau_{1}$ and $\tau_{2}$ are depicted on Figure 8 (a)-(b). Some comments arise: (i) In case with torque $\tau_{1}$, the revolute joint $J_{3}$ with clearance $c_{3}$ has more influence on the system's performance as compared to joint $J_{2}$ with clearance $c_{2}$. This may be attributed to the location of the applied torque. (ii) As expected the torque $\tau_{2}$ yields unpredictable behaviour with high sensitivity of $e\left(c_{2}, c_{3}\right)$ (Figure $8(\mathrm{~b})$ ). We infer from Figures 4 (b) and 8 (b) that the system actuated with $\tau_{2}$ is quite sensitive to both initial data and clearances values. The simulations for Figure 8 (b) were led over $[0,100] \mathrm{s}$ in order to capture the long-term behaviour of the trajectories (as seen on Figures 6 (c) and (d) 


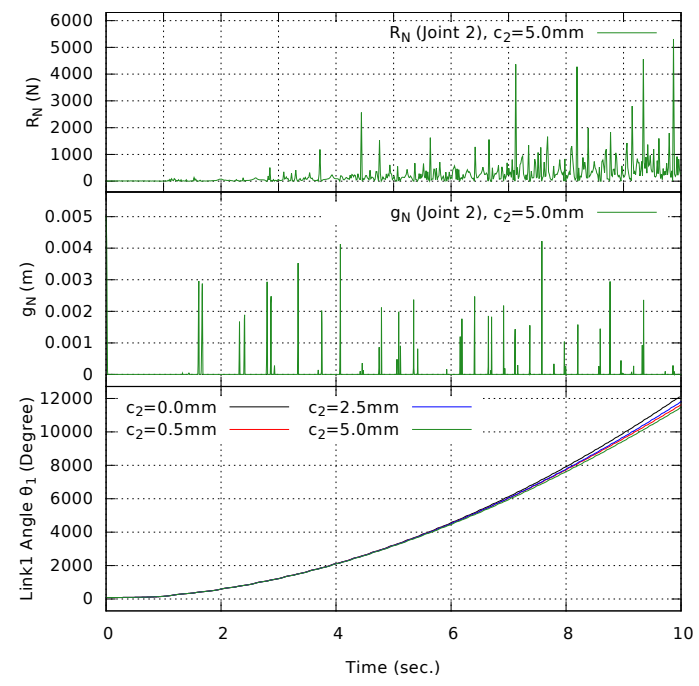

(a) Without friction, $\mu=0.0, \tau=\tau_{1}$.

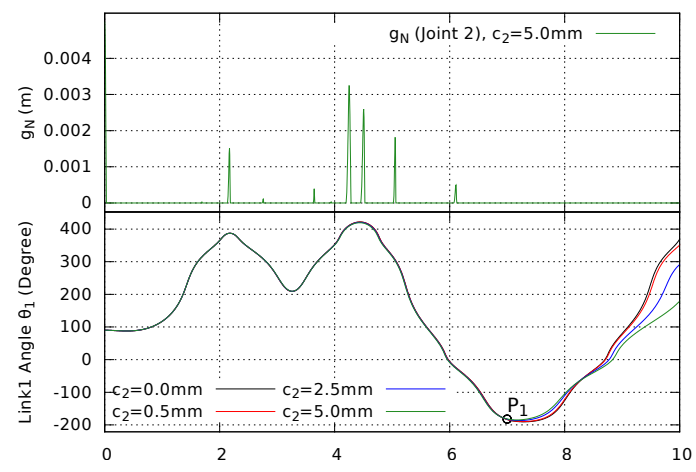

(c) Zoomed on $T \in[0,10] \mathrm{s}, \mu=0.0, \tau=\tau_{2}$.

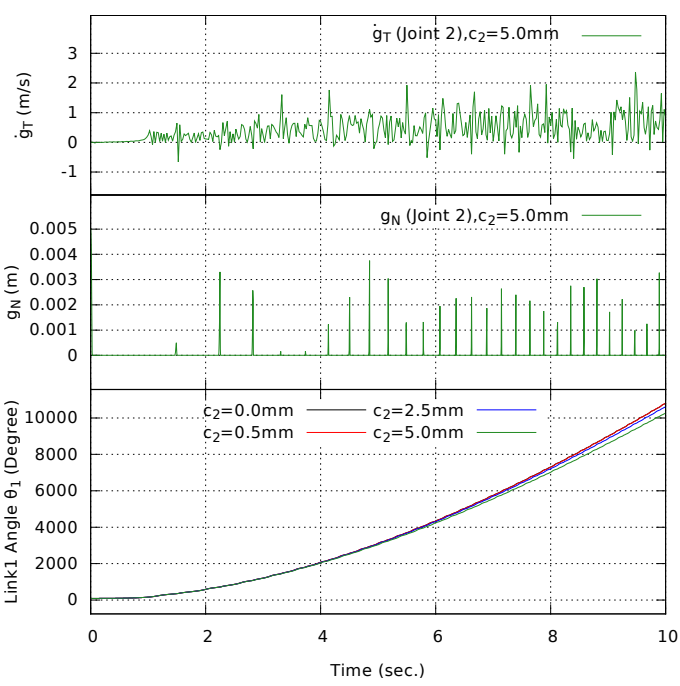

(b) With friction, $\mu=0.1, \tau=\tau_{1}$.

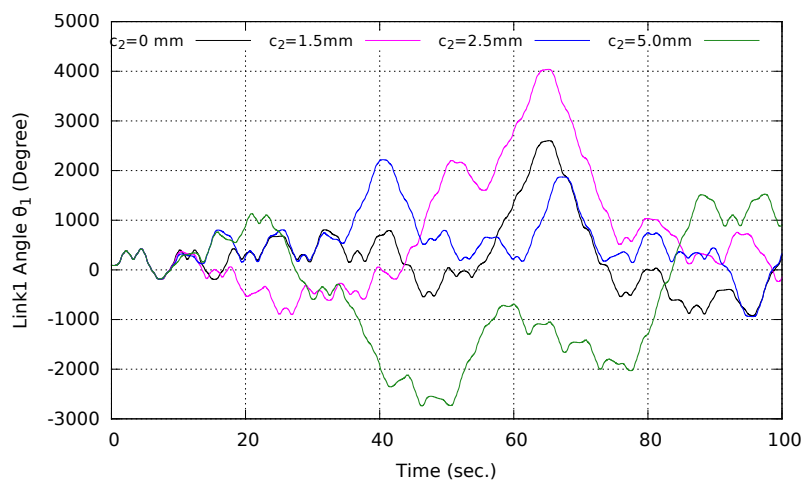

(d) Without friction, $\mu=0.0, \tau=\tau_{2}$

Figure 6: Crank-rocker with clearance in $J_{2}: \theta_{1}, g_{N}, \dot{g}_{T}$ and $R_{N}, e_{r}=0.0$.

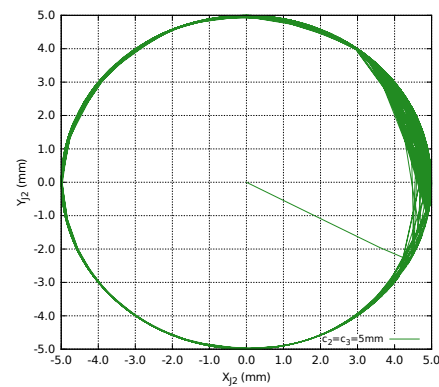

(a) $e_{r}=0.0, t \in[0,10] \mathrm{s}$.

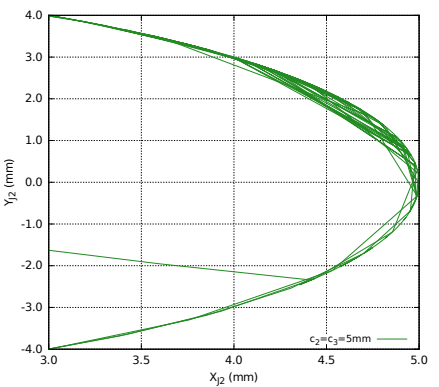

(b) $e_{r}=0, t \in[0,3] \mathrm{s}$.

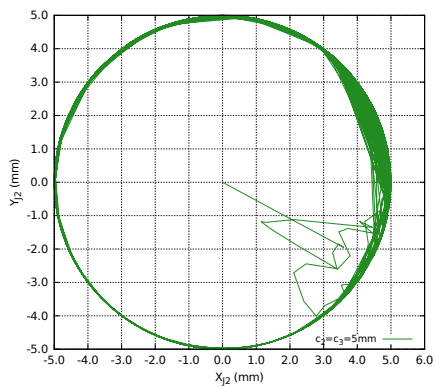

(c) $e_{r}=0.9, t \in[0,10] \mathrm{s}$.

Figure 7: Crank-rocker with clearance in $J_{2}$ : Journal center locus, $\tau_{1}$.

with $c_{3}=0$, trajectories with and without clearance remain close one to each other for $\tau_{2}$ on the first $10 \mathrm{~s})$. 


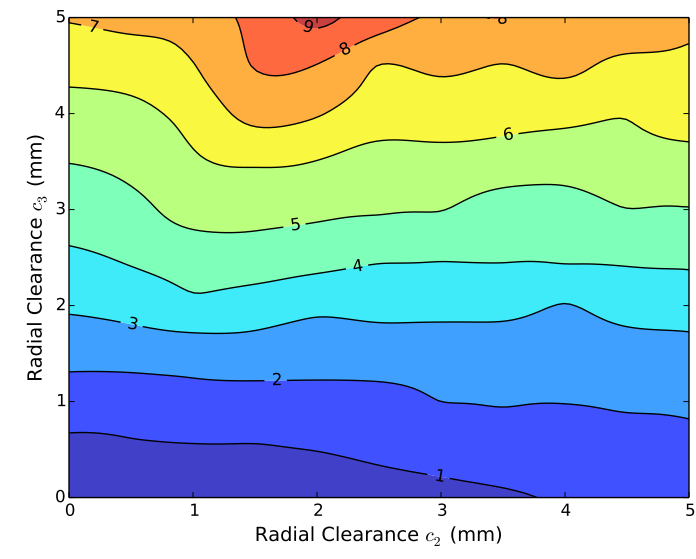

(a) Torque $\tau_{1}$.

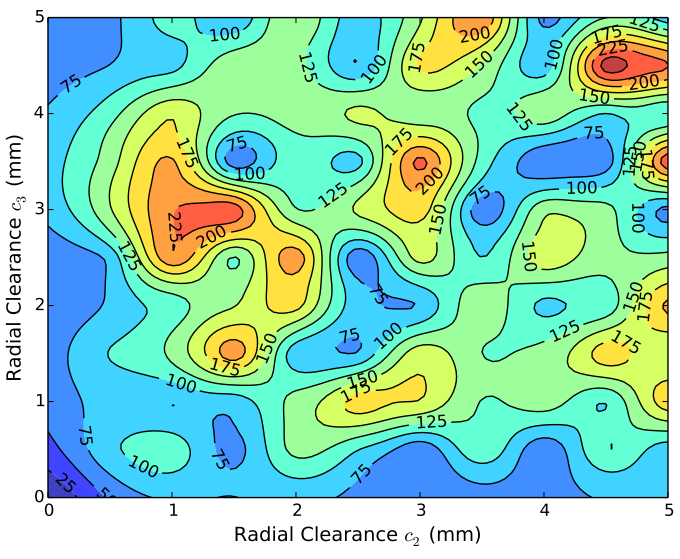

(b) Torque $\tau_{2}$.

Figure 8: Crank-rocker with clearance in $J_{2}, J_{3}$ : contour plot of $e$, with $e_{r}=0.0, \mu=0.1$.

\section{State-feedback control}

The main conclusion from the foregoing section is that open-loop controllers may easily lead to unpredictable behaviour with high sensitivity to both initial data and clearance values, when non-constant torques are applied. With such a high sensitivity, it is hopeless to try to deduce some universival conclusions on the relative influence of the parameters $\left(e_{r}, \mu, c_{2}, c_{3}\right)$ on the behavior of the mechanism. It is of interest to investigate if adding a collocated feedback action at joint $J_{1}$ may improve the system's dynamical behaviour when clearances are present (the answer for the no-play case being trivially positive in case of the two nonlinear controllers which guarantee global exponential Lyapunov stability of the tracking error system). We will in the following consider four types of feedback controllers with increasing complexity: proportional-derivative (PD) plus gravity compensation, with and without desired velocity, feedback linearization, and passivity-based inputs. There are many other types of controllers that have been derived for Lagrangian systems, starting from the basic PD and PID controllers, see e.g. [60, 33, 4, 34, 9]. In this study we chose to focus on few of them only, for obvious reasons.

\subsection{Proportional-Derivative (PD) controllers}

In this section two different types of PD controllers are considered:

$$
\tau_{3}\left(\theta_{1}, \dot{\theta}_{1}, t\right)=-K_{2} \dot{\theta}_{1}-K_{1}\left(\theta_{1}-\theta_{1}^{d}(t)\right)
$$

and

$$
\tau_{4}\left(\theta_{1}, \dot{\theta}_{1}, t\right)=-K_{2}\left(\dot{\theta}_{1}-\dot{\theta}_{1}^{d}(t)\right)-K_{1}\left(\theta_{1}-\theta_{1}^{d}(t)\right)
$$

where $K_{1}$ and $K_{2}$ are positive control gains.

Since the system in (8) is non-linear, PD controllers without any kind of feedforward compensation do not a priori guarantee the global asymptotic trajectory tracking of the dynamics (37) with (17) or (18). However the input $\tau_{4}$ guarantees the global practical stability [9, Theorem 1]. The choice of the gains may be made by varying the gains and computing the maximum tracking error $\tilde{\theta}_{1} \triangleq \theta_{1}-\theta_{1}^{d}$ in each case, where the desired angle has been chosen as $\theta_{1}^{d}(t)=6.0 \sin (0.75 \pi t)$ for the crank-rocker and the crank- 
crank, $\theta_{1}^{d}(t)=3.0+2.5 \sin (0.75 \pi t)$ for the rocker-rocker mechanisms. The maximum tracking errors on $[0,10] \mathrm{s}$ for the crank-rocker, crank-crank and rocker-rocker four-bar mechanisms are plotted for different values of the control gains $K_{1}$ and $K_{2}$ on Figure 9. As expected from [9, Theorem 1], the tracking error decreases as $K_{1}$ and $K_{2}$ increase, and quickly attains an almost constant value for the three mechanisms and both controllers. It is interesting to note that the crank-crank mechanism shows the largest tracking error: this may be due to the fact that the nonlinear torque $N\left(\theta_{1}, \dot{\theta}_{1}\right)$ in 37 has bigger magnitude than for the other two mechanisms. Also the input $\tau_{4}$ permits to decrease significantly the tracking error for large enough gains, while $\tau_{3}$ cannot: this demonstrates the usefulness of the feedforward velocity term $K_{2} \dot{\theta}_{1}^{d}$ in 18 . For the sake of comparison between the various feedback controllers these gains will also be used for the PD-part of the nonlinear inputs of Sections 4.2 and 4.3 . Thus they have to satisfy the conditions stated in Appendix D. The choice has been made as $K_{1}=2000$ and $K_{2}=200$, because larger values do not improve the performance as shown on Figure 9. The constant $C$ in the Lyapunov function 42) can be chosen $C=10$.

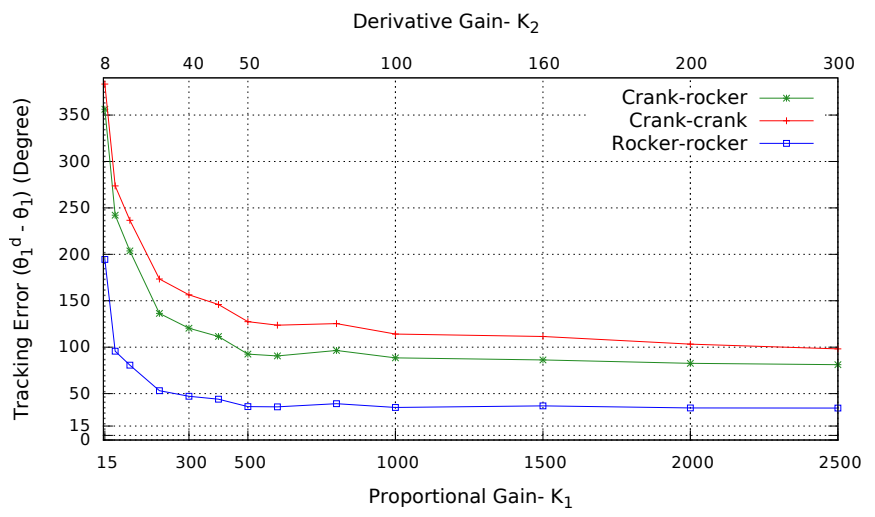

(a) $\tau=\tau_{3}$.

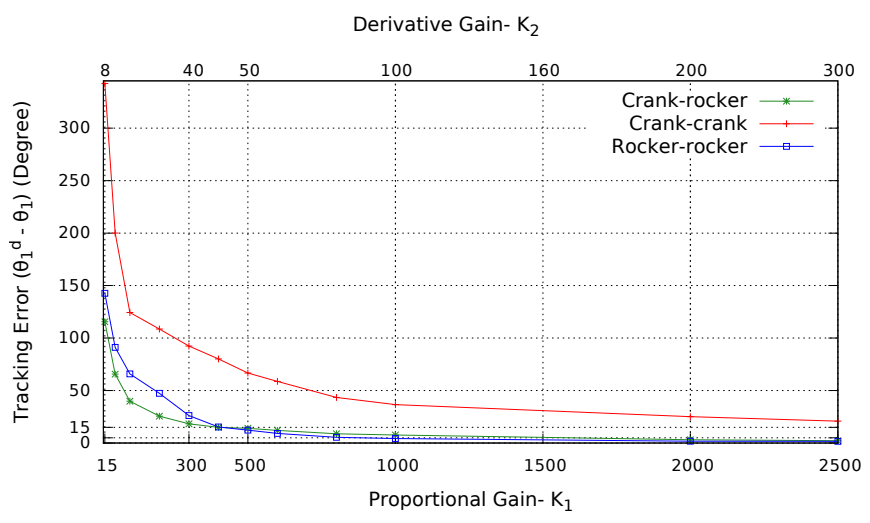

(b) $\tau=\tau_{4}$.

Figure 9: PD control: maximum tracking error $\theta_{1}^{d}(t)-\theta_{1}(t) v s$. controller gains.

\subsubsection{Crank-rocker mechanism}

Let us consider a crank-rocker mechanism with clearance in one and two revolute joints (see Figures 1(a) and 3(a)-(b)). The Lagrange dynamics is given as in Appendices B and C, respectively, and the system is underactuated with collocated input at joint $J_{1}$. The geometric and inertial properties, parameters used for simulation and initial conditions are given in Section 3. The isolines of $e$ in (16) which allow us to compare the cases with and without clearances, are depicted on Figure 10 . They were found to be identical for both $\tau_{3}$ and $\tau_{4}$, which shows that the addition of $\dot{\theta}_{1}^{d}(t)$ in $\tau_{4}$ may improve the tracking capabilities, while the system's precision deterioration is unchanged when clearances are added. Only one set of simulations is shown because changing $e_{r}$ and $\mu$ did not change the results significantly. Comparing Figures 8 (b) and 10 (b) shows a significant discrepancy between open-loop and state feedback controllers. Actually, the Lyapunov stability of closed-loop systems with state feedback controllers, drastically changes their dynamical behaviour when clearances are present. It is noteworthy that the coefficient of restitution plays no role in the variation of $e$ (see Figure 10(a)), and there exsist a symmetry ofthe behaviour with respect to clearances $c_{2}$ and $c_{3}$ (see Figure 10 (b)). From Figure 12 we conclude that, similarly to the case of input $\tau_{1}$, the journal spends most of the time almost in contact with the 
bearing, with very small rebounds excepted in few cases where the journal crosses the whole bearing, when the desired trajectory changes its direction (see Figure 12 (b)).

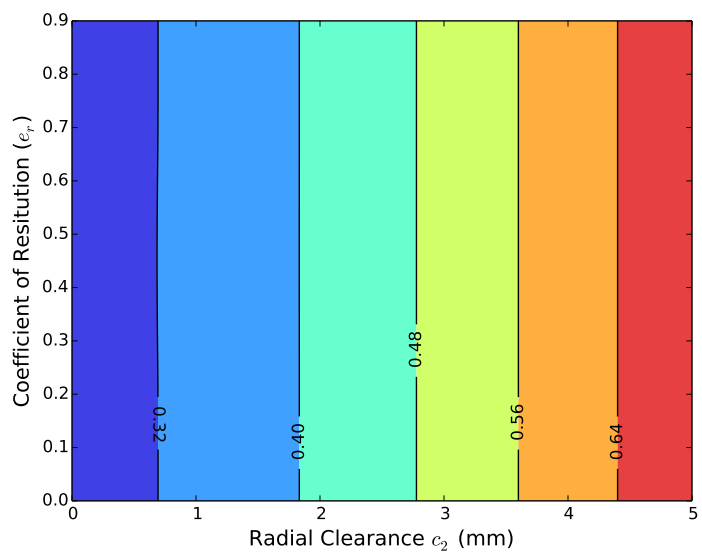

(a) Clearance in $J_{2}: e$ with $c_{2}$ and $e_{r}$.

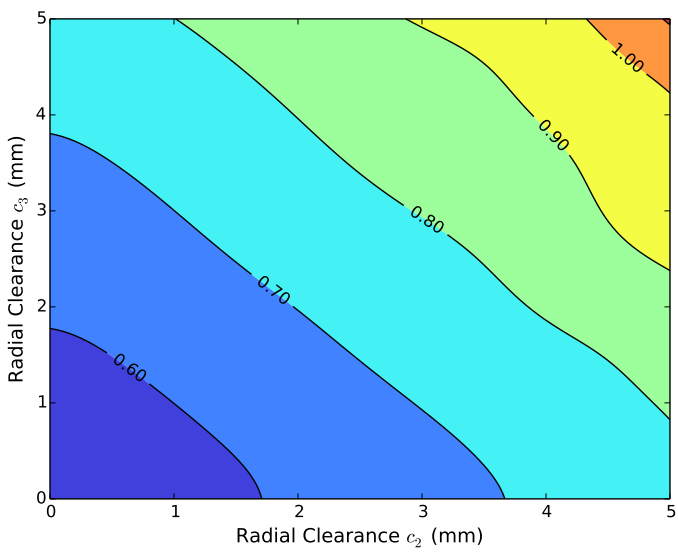

(b) Clearance in $J_{2}, J_{3}: e$ with $c_{2}$ and $c_{3}, e_{r}=0.0$.

Figure 10: Crank-rocker with PD control: contour plot of $e, \mu \in\{0.0,0.1\}, \tau_{3}$ and $\tau_{4}$.

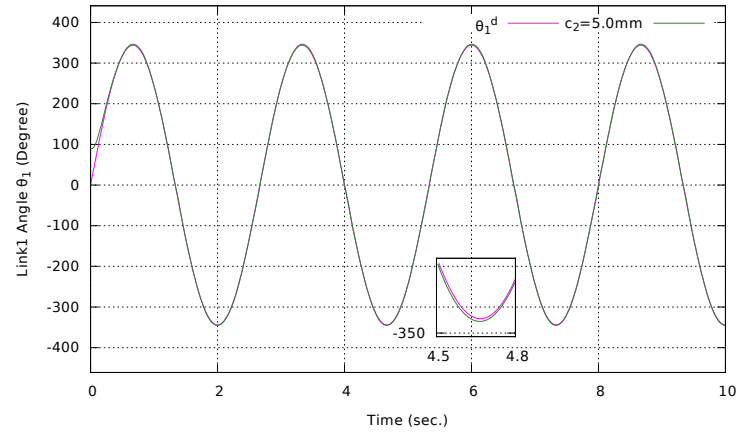

(a) Clearance in one joint $J_{2}, \theta_{1}(t)$ and $\theta_{1}^{d}(t)$.

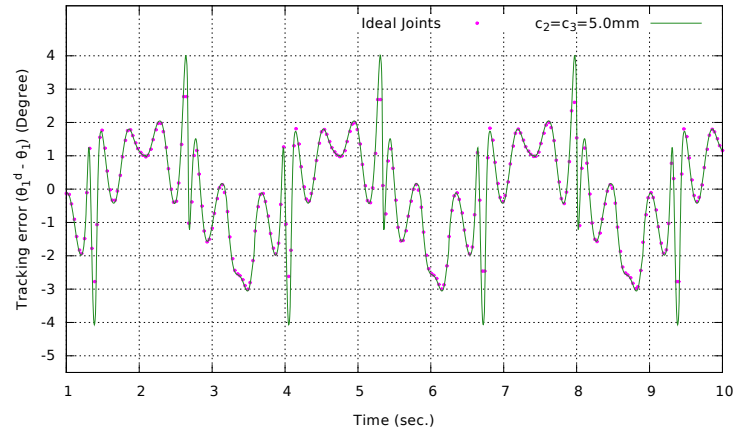

(b) Tracking error $\tilde{\theta}_{1}$.

Figure 11: Crank-rocker with PD control: $\theta_{1}$ and $\tilde{\theta}_{1}\left(e_{r}=0.0, \mu=0.1, \tau=\tau_{4}\right)$.

\subsubsection{Crank-crank and rocker-rocker mechanisms}

Let us consider a crank-crank mechanism with clearance in one and two revolute joints (see Figures 1.(b) and 3(a)-(b)). The geometric and inertial properties are given in Table 3. The control gains are

Table 3: Geometric and inertial properties of the crank-crank four-bar mechanism.

\begin{tabular}{cccc}
\hline \hline Body Nr. & Length $[\mathrm{m}]$ & Mass $[\mathrm{kg}]$ & Inertia $\left[\mathrm{kg} \mathrm{m}^{2}\right]$ \\
\hline 1 & 1.2 & 1.0 & $1.20 \cdot 10^{-1}$ \\
2 & 1.2 & 1.0 & $1.20 \cdot 10^{-1}$ \\
3 & 1.2 & 1.0 & $1.20 \cdot 10^{-1}$ \\
4 & 1.0 & - & -
\end{tabular}

unchanged. The initial conditions are $\theta_{1}(0)=1.658 \mathrm{rad}, \theta_{2}(0)=1.607 \cdot 10^{-4} \mathrm{rad}, \theta_{3}(0)=1.488 \mathrm{rad}$, $\dot{\theta}_{1}(0)=\dot{\theta}_{2}(0)=\dot{\theta}_{3}(0)=0.0 \mathrm{rad} / \mathrm{s}$. The control performance are depicted on Figures 13 and 14 . The counterparts of Figures 10 and 13 for the rocker-rocker mechanism are not shown because they are quite 


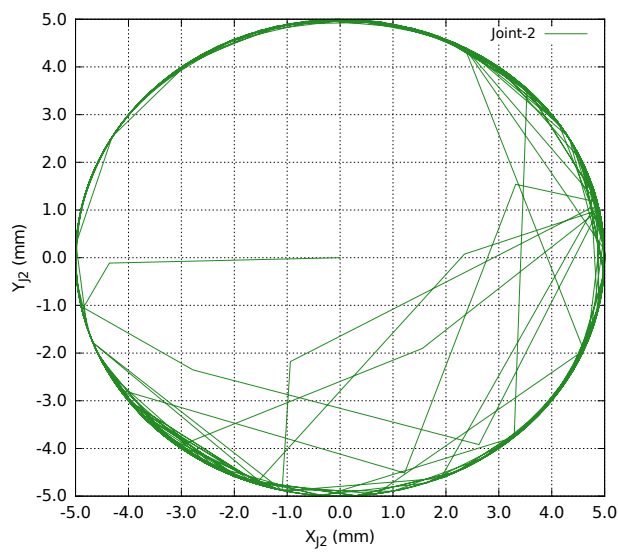

(a) $T \in[0,10] \mathrm{s}$.

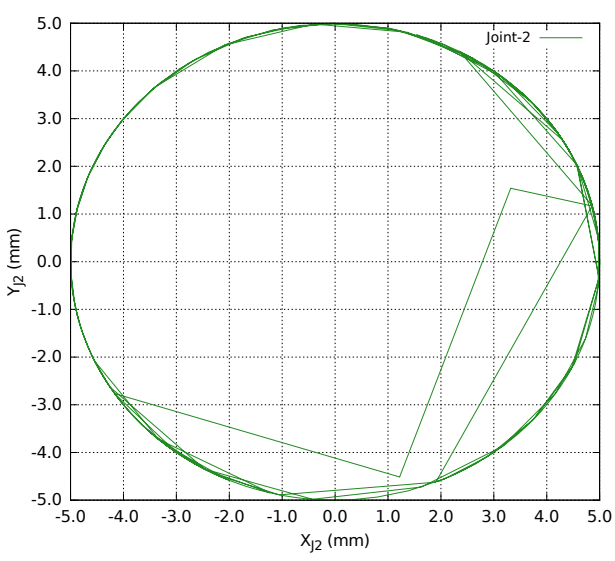

(b) $T \in[5.4,8.0] \mathrm{s}$

Figure 12: Crank-rocker with PD control: journal locus inside the bearing.

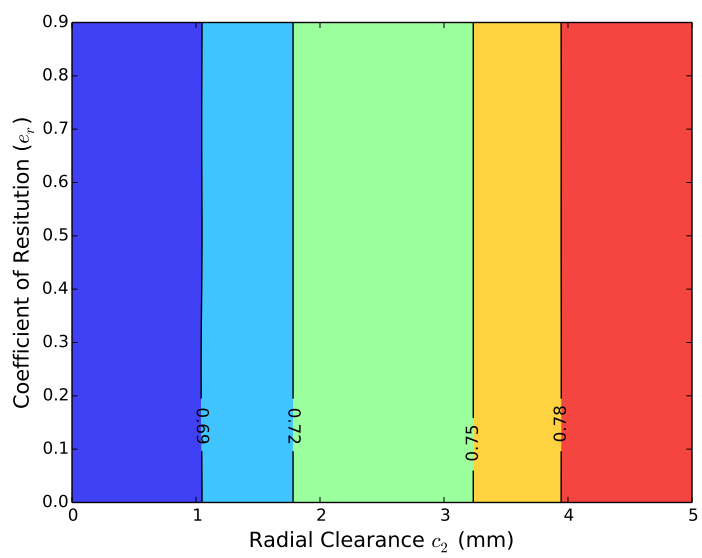

(a) Clearance in $J_{2}: e$ with $c_{2}$ and $e_{r}$.

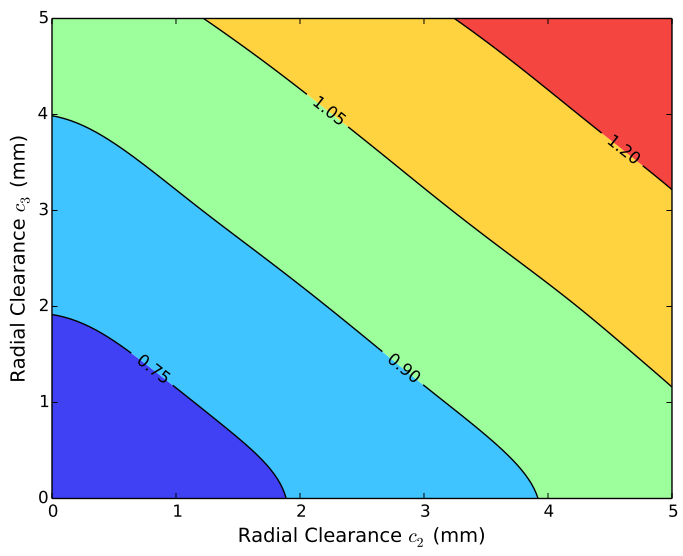

(b) Clearance in $J_{2}, J_{3}: e$ with $c_{2}$ and $c_{3}, e_{r}=0.0$.

Figure 13: Crank-crank with PD control: contour plot of $e, \mu \in\{0.0,0.1\}, \tau_{3}$ and $\tau_{4}$.

similar to the other two.

\subsubsection{Conclusion on PD control}

It is visible on Figures 10 and 13 that (i) the closed-loop behaviour of both PD controllers in (17) and (18) is predictable (the restitution coefficient $e_{r}$ has negligible influence on $e$, while a symmetric influence of $c_{2}$ and $c_{3}$ is observed), (ii) the values of $e$ are however much smaller than those for $\tau_{1}$, indicating that the PD feedback has a significant influence on the system's dynamics in the presence of clearances, (iii) the tracking error is decreasing when $\tau_{4}$ is used instead of $\tau_{3}$ (see Figure 9) however this has little influence on $e$ : both controllers gave the same results on Figures 10 and 13 , (iv) from Figures 9 10, 11, 13 and 14 it follows that the crank-rocker mechanism provides better performance than the crankcrank one, both for $e$ and the precision at the velocity sign changes (see the zoomed parts on Figures 11 (a) and 14), (v) as expected the loss of precision occurs when the desired trajectory changes direction 


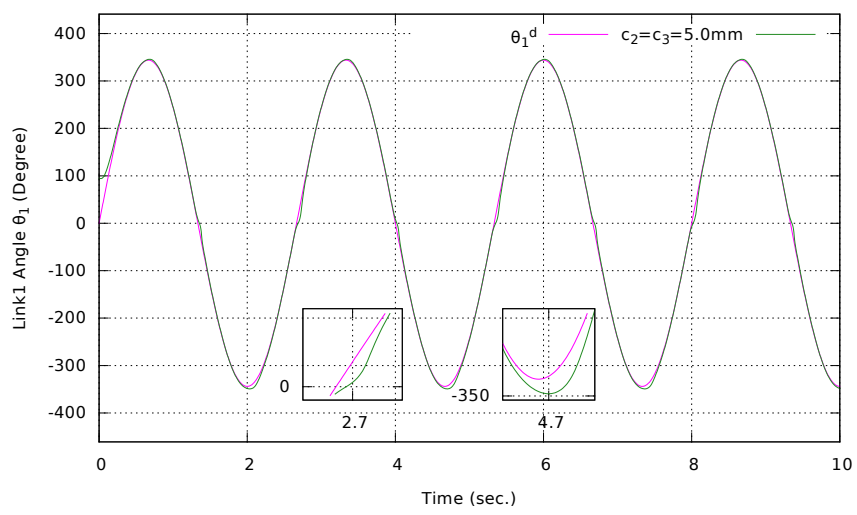

Figure 14: Crank-crank: PD control with clearance in two joints $\left(J_{2}, J_{3}\right): \theta_{1}\left(e_{r}=0.0, \mu=0.1, \tau=\tau_{4}\right)$.

(see Figure 14). This is what motivated some extensions of the PD controllers to improve the accuracy [32].

\subsection{State feedback linearization}

The smooth part of the dynamic equations of the four-bar mechanism with minimal coordinate is:

$$
M\left(\theta_{1}\right) \ddot{\theta}_{1}+N\left(\theta_{1}, \dot{\theta}_{1}\right)+g\left(\theta_{1}\right)=\tau_{5}
$$

Details on how to obtain this minimal coordinate dynamics are given in Appendix A Let us choose the control torque as:

$$
\tau_{5}\left(\theta_{1}, \dot{\theta}_{1}, U\right)=M\left(\theta_{1}\right) U+N\left(\theta_{1}, \dot{\theta}_{1}\right)+g\left(\theta_{1}\right)
$$

The control law (20) is a simple instance of state feedback linearization. Since $M\left(\theta_{1}\right)>0$, the closedloop system $(19)-(20)$ reduces to the double-integrator $\ddot{\theta}_{1}=U$. The input $U$ is chosen as PD controller $U\left(\theta_{1}, \dot{\theta}_{1}, t\right)=-K_{1} \theta_{1}-K_{2} \dot{\theta}_{1}+\mathbf{r}(t)$. For a given desired trajectory $\left(\theta_{1}^{d}(t), \dot{\theta}_{1}^{d}(t)\right)$ one sets $\mathbf{r}(t)=$ $\ddot{\theta}_{1}^{d}(t)+K_{2} \dot{\theta}_{1}^{d}(t)+K_{1} \theta_{1}^{d}(t)$. Then the tracking error satisfies the closed-loop dynamics :

$$
\left(\ddot{\theta}_{1}-\ddot{\theta}_{1}^{d}(t)\right)+K_{2}\left(\dot{\theta}_{1}-\dot{\theta}_{1}^{d}(t)\right)+K_{1}\left(\theta_{1}-\theta_{1}^{d}(t)\right)=0
$$

which is globally exponentially stable, with a convergence speed depending on the choice of the controller gains. The controller gains have to satisfy the conditions stated in Appendix D. Since the controller may be seen as a PD input with some nonlinearities compensation, the gains will be chosen as for the PD controllers $K_{1}=2000$ and $K_{2}=200$ for the sake of comparison.

For the sake of brievity and since the results we obtained were quite similar for the three mechanisms, we shall consider in this section a crank-rocker mechanism with clearance in one and two revolute joints (see Figures 1 (a) and 3(a)-(b)). The desired trajectory of the input link is given as $\theta_{1}^{d}=6.0 \sin (0.75 \pi t)$. The geometric and inertial properties, parameters used for simulation are given in Tables 1. 2, the initial conditions are as in Section 3. $\theta_{1}(0)=1.571 \mathrm{rad}, \theta_{2}(0)=0.3533 \mathrm{rad}$, $\theta_{3}(0)=1.2649 \mathrm{rad}, \dot{\theta}_{1}(0)=\dot{\theta}_{2}=\dot{\theta}_{3}(0)=0.0 \mathrm{rad} / \mathrm{s}$. The numerical simulations are depicted on Figures 15, 16 and 17 for the case with clearances in one and two revolute joints. On Figure 16, the trajectories of the input link $\theta_{1}(t)$ for various clearances, as well as the Lyapunov function $V(z)$ in 42 . are shown. The results have been obtained for different values of $e_{r} \in[0.0,0.9]$ and for two different values of $\mu=0.0$ and $\mu=0.1$. However only one set of simulation is shown because changing $e_{r}$ and 
$\mu$ did not change the results significantly. Some comments arise: (i) Compared to the PD controller, the

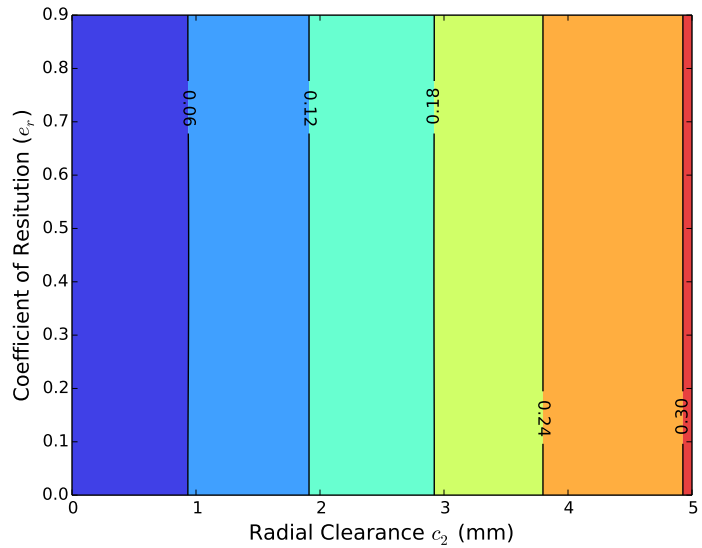

(a) Clearance in $J_{2}: e$ with $c_{2}$ and $e_{r}$.

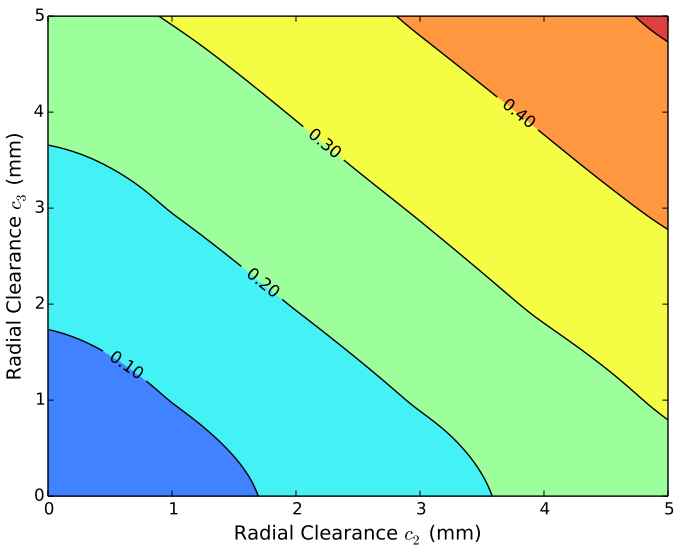

(b) Clearance in $J_{2}, J_{3}: e$ with $c_{2}$ and $c_{3}, e_{r}=0.0$.

Figure 15: Crank-rocker with state linearization control: contour plot of $e, \mu \in\{0.0,0.1\}, \tau=\tau_{5}$.

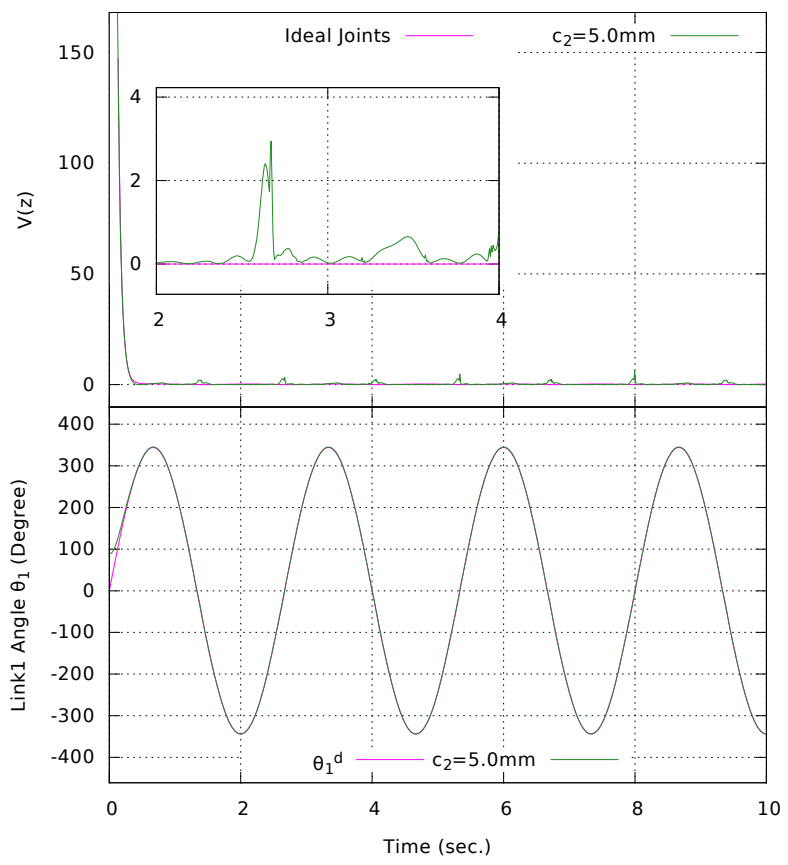

(a) Clearance in one joint $J_{2}$.

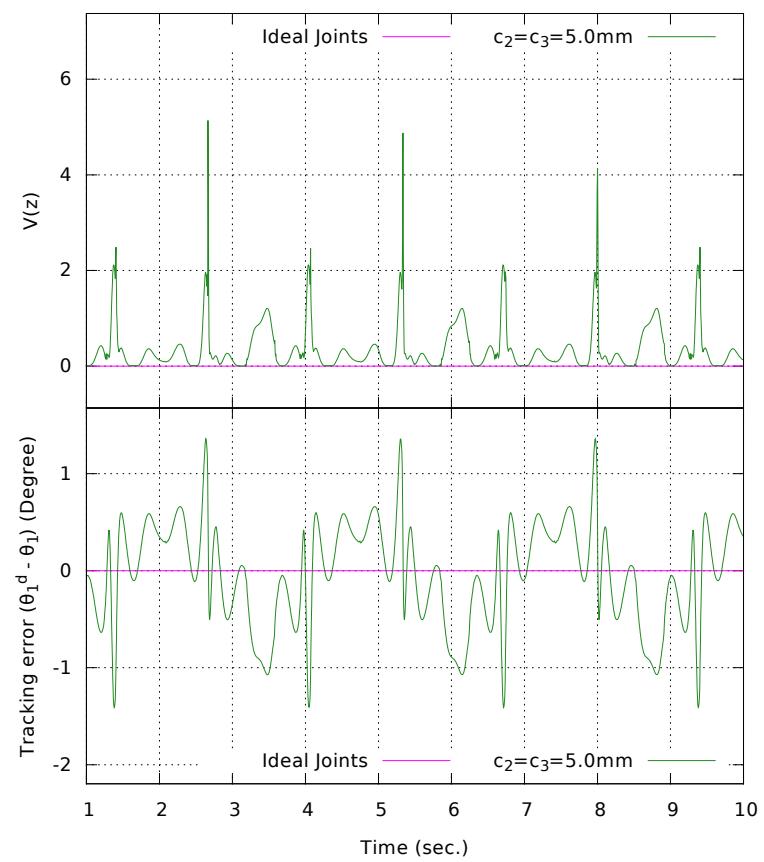

(b) Clearance in two joints $J_{2}$ and $J_{3}$.

Figure 16: Crank-rocker with state linearization control: $\theta_{1}, \tilde{\theta}_{1}$ and $V(z)\left(e_{r}=0.0, \mu=0.1, \tau=\tau_{5}\right)$.

error $e$ is smaller by a factor 2 for large clearances and a factor 5 for small clearances (see Figures 10 and 15). This tends to indicate that the feedback action and the compensation of nonlinearities both have a significant influence in the dynamics with play. (ii) The Lyapunov function shows persistent variations after an initial exponential decrease, see Figure 16. this is due to the impacts which make the velocity jump, and thus induce state re-initializations all along the system's motion. It is however a tough task 


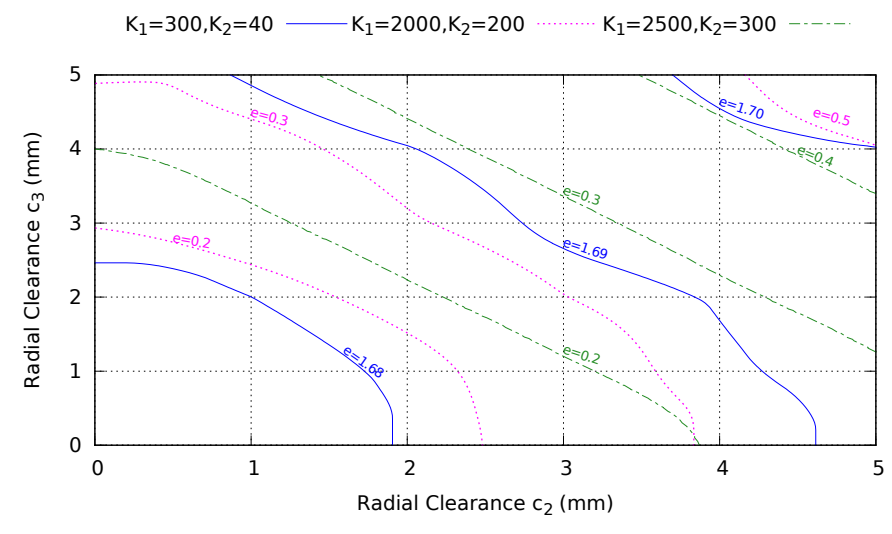

(a) Influence of control gain on $e, \tau_{5}$.

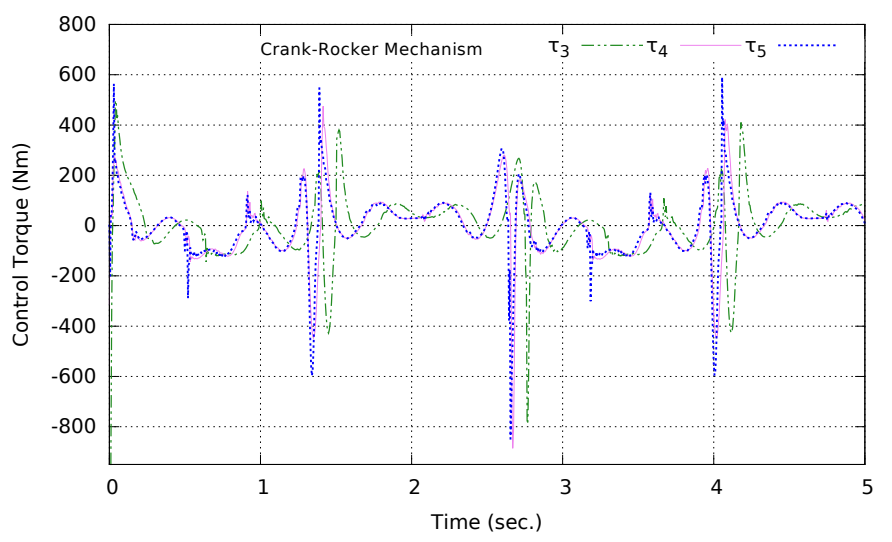

(b) Comparison: $\tau_{3}, \tau_{4}$ and $\tau_{5}$.

Figure 17: Crank-rocker with state linearization control.

to analyze conditions under which $V(z)$ remains bounded despite of impacts, because it involves an interplay between the positive jumps at impact times and the exponential decrease between impacts (while persistent contact phases of motion should also be taken into account in a theoretical analysis). (iii) The tracking error is reduced compared to the PD control, since $\tilde{\theta}_{1} \in[-4,4]$ for $\tau_{4}$ while $\tilde{\theta}_{1} \in[-1.3,1.3]$ for $\tau_{5}$ (see Figures 16 (b) and 11 (b)). Also $\tilde{\theta}_{1}$ with one clearance is smaller than with two clearances, compare $V(z)$ on Figures 16 (a) and (b). (iv) Increasing the gains $K_{1}$ and $K_{2}$ allows one to consider larger pairs of clearances $\left(c_{2}, c_{3}\right)$ for the same error $e$, as shown on Figure 17 (a). (v) The controllers $\tau_{3}$, $\tau_{4}$ and $\tau_{5}$ possess quite similar shapes and magnitudes, as depicted on Figure 17(b). However $\tau_{3}$ and $\tau_{4}$ take larger values during the transient period. The absence of feedforward term in $\tau_{3}$ induces a delay in its reaction to impacts, but $\tau_{4}$ behaves surprisingly close to the state feedback linearization scheme.

It is visible from Figure 15 that the three mechanisms, when controlled with a state feedback linearization algorithm, behave in the same way.

\subsubsection{Conclusions}

The feedback linearization control schemes clearly supersede the PD controllers both from the point of views of tracking error reduction (which is a well-known result) but also for the error $e$ reduction. The second set of results (Figures 10, 13 and 15) means that compensation of the smooth nonlinearities allows to reduce the closed-loop system's sensitivity w.r.t. the presence of clearances.

\subsection{Passivity-based control}

Passivity-based controllers have become quite popular for the control of nonlinear mechanical systems [8]. Let us investigate now the behaviour of the so-called Slotine and Li controller with fixed parameters, which is given in the no-clearance case (37) as:

$$
\left\{\begin{array}{l}
\tau_{6}\left(\theta_{1}, \dot{\theta}_{1}, t\right)=M\left(\theta_{1}\right)\left(\ddot{\theta}_{1}^{d}(t)-\Lambda\left(\dot{\theta}_{1}-\dot{\theta}_{1}^{d}(t)\right)\right)+C\left(\theta_{1}, \dot{\theta}_{1}\right)\left(\dot{\theta}_{1}^{d}-\Lambda\left(\theta_{1}-\theta_{1}^{d}(t)\right)\right)+g\left(\theta_{1}\right)-K v \\
v=\left(\dot{\theta}_{1}-\dot{\theta}_{1}^{d}(t)\right)+\Lambda\left(\theta_{1}-\theta_{1}^{d}(t)\right)
\end{array}\right.
$$

where $C\left(\theta_{1}, \dot{\theta}_{1}\right) \dot{\theta}_{1}=N\left(\theta_{1}, \dot{\theta}_{1}\right)$. The control gain $K$ is similar to the derivative control gain $K_{2}$ and the control gain $K \Lambda$ is similar to the proportional control gain $K_{1}$. Thus the control gains are chosen as: $K=200$ and $\Lambda=10$. The closed-loop dynamics 22 37) reads as $M\left(\theta_{1}\right) \dot{v}+C\left(\theta_{1}, \dot{\theta}_{1}\right) v+K v=0$, 
and $\dot{\tilde{\theta}}_{1}=-\Lambda \tilde{\theta}_{1}+v$.

\subsubsection{Collocated control of crank-rocker mechanism}

Once again for the sake of brievity we shall consider in this section a crank-rocker mechanism only. The geometric and inertial properties, parameters used for simulation and initial conditions are as above. The numerical simulations are depicted in Figures 18, 19, 20 and 21, and in Tables 4 and 5, The results have been obtained for different values of $e_{r} \in[0.0,0.9]$ and for two different values of $\mu=0.0$ and $\mu=0.1$. However only one set of simulation is shown because changing $e_{r}$ and $\mu$ did not change the results significantly. Some comments are as follows: (i) Figures 15 and 18 (a)-(b)) show that the

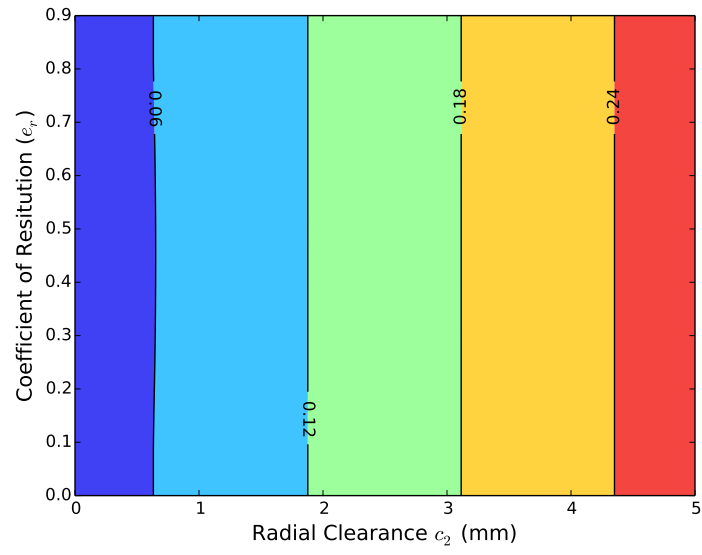

(a) Clearance in $J_{2}: e$ with $c_{2}$ and $e_{r}$.

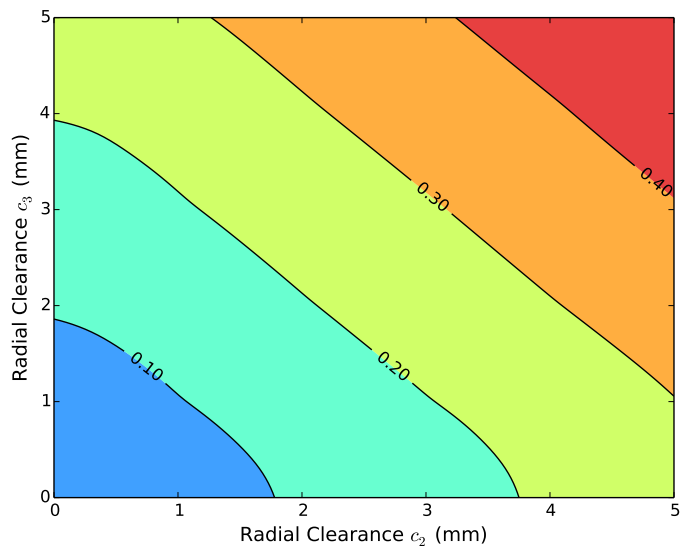

(b) Clearance in $J_{2}, J_{3}: e$ with $c_{2}$ and $c_{3}, e_{r}=0.0$.

Figure 18: Crank-rocker with passivity-based control: contour plot of $e, \mu \in\{0.0,0.1\}, \tau=\tau_{6}$.

passivity-based control algorithm is slightly less sensitive to the clearances than the state linearization one. However the tracking errors are similar for both controllers (see Figures 16 (b), 19 (b) and Tables 4. 5. 6. (ii) For the same precision, the control torque has smaller peaks magnitude when compared to feedback linearization, as shown on Figure 20 and in Table 4 for various gains. (iii) When the gains are decreased, the maximum tracking error remains almost identical for both controllers, but the passivitybased input maximum value decreases much more than that of the state linearization input (see Table 4). This may be explained by the fact that passivity-based controllers do not totally compensate the Lagrange dynamics nonlinearities, and thus induce less solicitation of the input torque. (iv) The evolution of the Lyapunov-like function $V(v)$ defined in (44) is depicted on Figure 19 (a) and (b). It shows that the case with one clearance has less impacts than two clearances (similarly to the state linearization on Figure 16), and it seems that some periodic nonsmooth motion exists in steady-state 5 (v) Figure 21 shows the typical behaviour inside a clearance ( $X_{j 2}$ and $Y_{j 2}$ denote the relative position of $O_{2}$ inside the bearing): there are few impacts and the system tends to evolve on the bearing's surface. This once again explains why for such desired trajectories, the restitution coefficient does not play a significant role. Comparing Figures 21 and 12 , we infer that compensating for smooth nonlinearities does not modify significantly the journal center's motion inside the bearing: most of the time the system evolves with small values of the gap function. (vi) The influence of the desired trajectory frequency is reported in Table 5. The

\footnotetext{
${ }^{5}$ Once again, proving such assertions is far from trivial and is not tackled here.
} 


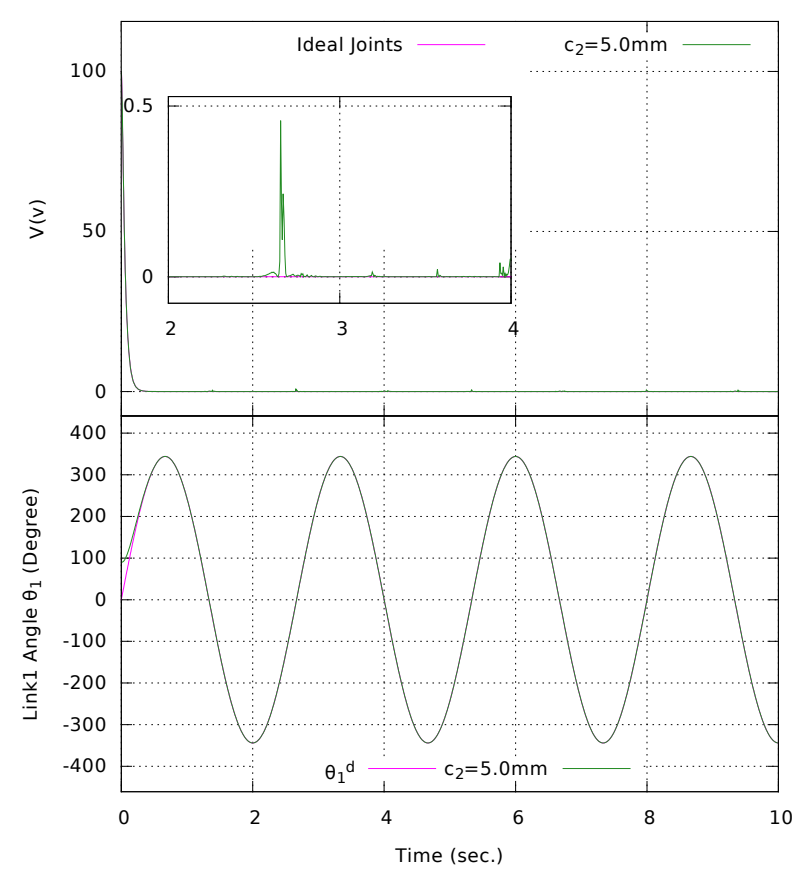

(a) Clearance in one joint $J_{2}$.

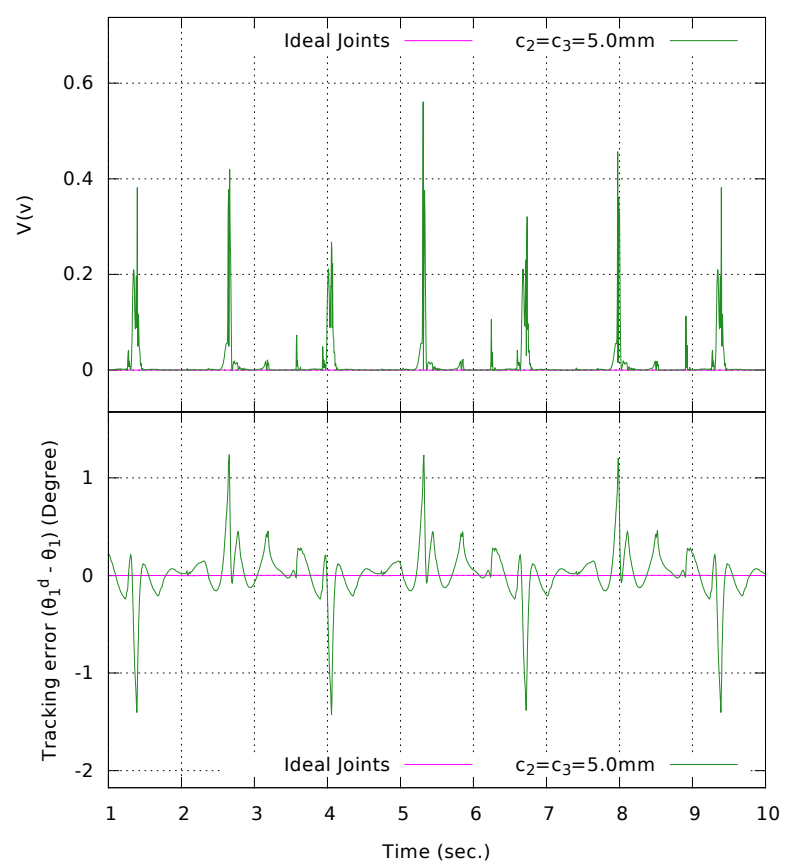

(b) Clearance in two joints $J_{2}$ and $J_{3}$.

Figure 19: Crank-rocker with passivity-based control: $\theta_{1}, V(v)$ and $\tilde{\theta}_{1}\left(e_{r}=0.0, \mu=0.1, \tau=\tau_{6}\right)$.

torques $\tau_{5}$ and $\tau_{6}$ show comparable behaviour when the frequency is increased. High frequencies induce large maximum tracking errors because the initial error $\dot{\tilde{\theta}}_{1}(0)$ is larger due to the larger desired velocity $\dot{\theta}_{1}^{d}(0)$.

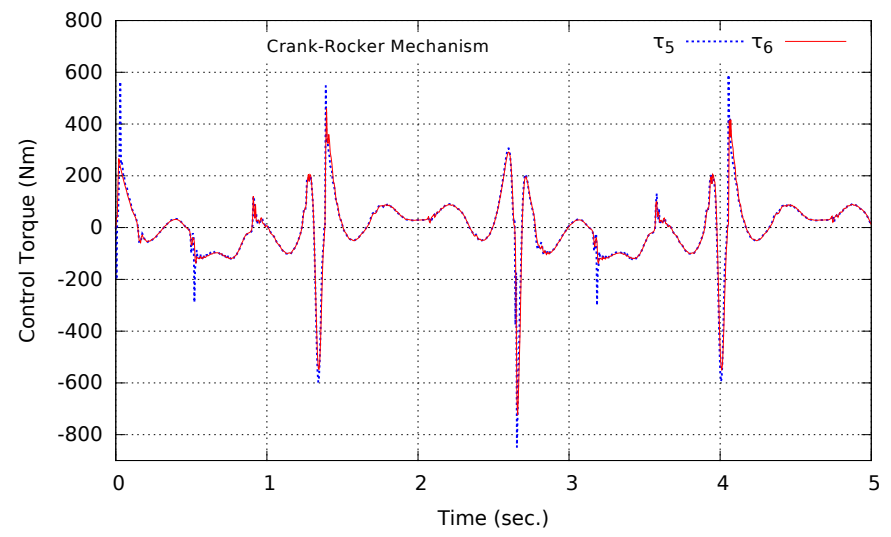

Figure 20: Crank-rocker: comparison of control torques $\tau_{5}$ and $\tau_{6}$.

Remark 3. (i) The contact/impact model has a great influence on the computed journal center motion inside the bearing [22] Figure 4.24]. As alluded to above, the model we chose together with the NSCD method of [2] allows to treat in a clean way the contact phases, avoiding non physical oscillations. Choosing compliant models would yield quite different journal center trajectories. 


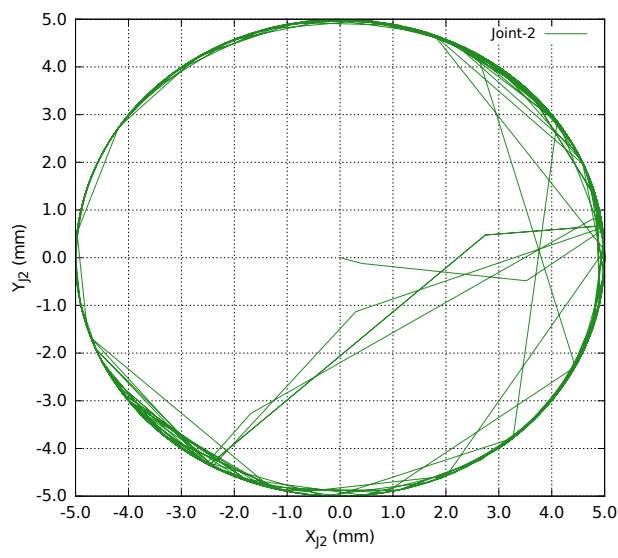

(a) Joint $J_{2}: T \in[0,10] \mathrm{s}$.

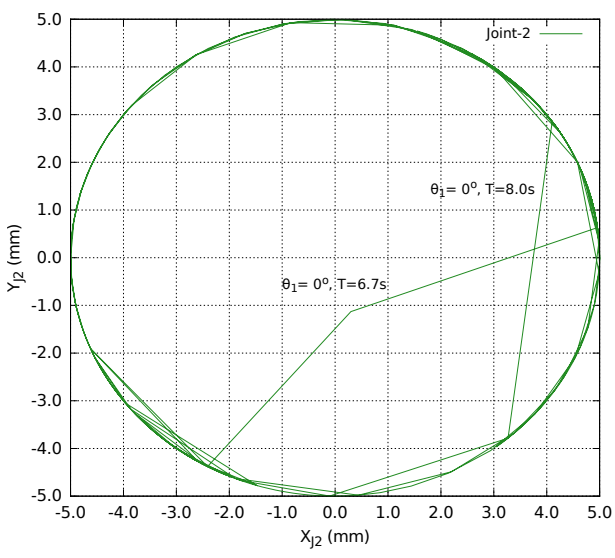

(b) Joint $J_{2}: T \in[5.4,8.0] \mathrm{s}$.

Figure 21: Crank-rocker with passivity-based control: journal center locus for joint 2.

Table 4: Crank-rocker: influence of control gains on the maximum tracking error on $[1,10] \mathrm{s}$ and control torque $\left(c_{2}=c_{3}=5.0 \mathrm{~mm}\right)$.

\begin{tabular}{|c|l|c|c|c|}
\hline Sr.No. & Type of controller & Control gain & $\begin{array}{c}\text { Max.Tracking } \\
\text { error (Degree) }\end{array}$ & $\begin{array}{c}\text { Max.Control } \\
\text { torque (Nm) }\end{array}$ \\
\hline \multirow{2}{*}{1} & $\begin{array}{l}\text { Feedback } \\
\text { Linearization } \tau_{5}\end{array}$ & $K_{1}=2000, K_{2}=200$ & 1.34 & 818.29 \\
\cline { 2 - 5 } & Passivity-based $\tau_{6}$ & $\Lambda=10, K=200$ & 1.3 & 724.45 \\
\hline \multirow{2}{*}{2} & $\begin{array}{l}\text { Feedback } \\
\text { Linearization } \tau_{5}\end{array}$ & $K_{1}=500, K_{2}=100$ & 2.94 & 782.59 \\
\cline { 2 - 5 } & Passivity-based $\tau_{6}$ & $\Lambda=5, K=100$ & 2.87 & 604.47 \\
\hline \multirow{2}{*}{3} & $\begin{array}{l}\text { Feedback } \\
\text { Linearization } \tau_{5}\end{array}$ & $K_{1}=100, K_{2}=50$ & 9.8 & 697.69 \\
\cline { 2 - 5 } & Passivity-based $\tau_{6}$ & $\Lambda=2, K=50$ & 9.21 & 511.39 \\
\hline
\end{tabular}

(ii) A nonlinear feedback controller is considered in [57 Equation (30)], and applied to a slider-crank mechanism. Contact is modelled with a compliant model. Numerical simulations show possible chaotic behaviour. It would be interesting to redo the analysis in this paper on the same slider-crank system, to investigate in which way the contact model may change the conclusions, and whether or not the above feedback controllers suppress or not the chaos.

\subsubsection{Non-collocated control of crank-rocker mechanism}

All the above results are for the collocated case, i.e. we apply the control torque at joint $J_{1}$ and we measure $\theta_{1}$ and $\dot{\theta}_{1}$. It is however possible to use the expressions in 27 ) in order to obtain functions $\theta_{1}\left(\theta_{3}\right)$ and $\dot{\theta}_{1}\left(\theta_{3}, \dot{\theta}_{3}\right)$. In the ideal case, using the direct measure of $\theta_{1}$ and $\dot{\theta}_{1}$ to compute $\tau_{6}$, or measuring $\theta_{3}$ and $\dot{\theta}_{3}$, then calculating $\theta_{1}\left(\theta_{3}\right)$ and $\dot{\theta}_{1}\left(\theta_{3}, \dot{\theta}_{3}\right)$ and using these expressions to compute a non-collocated input $\tau_{7}$, strictly provide the same results because $\tau_{7}\left(\theta_{1}\left(\theta_{3}\right), \dot{\theta}_{1}\left(\theta_{3}, \dot{\theta}_{3}\right)\right)=\tau_{6}\left(\theta_{1}, \dot{\theta}_{1}\right)$. When clearances are present in joints $J_{2}$ and/or $J_{3}$, then $\tau_{7}$ and $\tau_{6}$ differ since the expressions $\theta_{1}\left(\theta_{3}\right)$ and $\dot{\theta}_{1}\left(\theta_{3}, \dot{\theta}_{3}\right)$ are no longer valid. It is well-known that non-collocation deteriorates the control performance, and may 
Table 5: Crank-rocker: influence of frequency on the maximum tracking error on $[1,10] \mathrm{s}$ and control torque.

\begin{tabular}{|c|c|c|c|c|c|c|c|c|}
\hline \multirow{2}{*}{ Frequency $(f)$} & \multicolumn{4}{|c|}{ Max. Tracking error (Degree) } & \multicolumn{3}{c|}{ Max. Control torque $(\mathrm{Nm})$} \\
\cline { 2 - 9 } & \multicolumn{2}{|c|}{ Ideal Joints } & \multicolumn{2}{c|}{$\begin{array}{c}\text { Clearance in } J_{2}, J_{3} \\
c_{2}=c_{3}=5.0 \mathrm{~mm}\end{array}$} & \multicolumn{2}{|c|}{ Ideal Joints } & \multicolumn{2}{c|}{$\begin{array}{c}\text { Clearance in } J_{2}, J_{3} \\
c_{2}=c_{3}=5.0 \mathrm{~mm}\end{array}$} \\
\cline { 2 - 9 } & $\tau_{5}$ & $\tau_{6}$ & $\tau_{5}$ & $\tau_{6}$ & $\tau_{5}$ & $\tau_{6}$ & $\tau_{5}$ & $\tau_{6}$ \\
\hline $1.5 \pi$ & 0.004 & 0.004 & 3.16 & 3.2 & $2.3 \cdot 10^{3}$ & $2.3 \cdot 10^{3}$ & $3.6 \cdot 10^{3}$ & $2.3 \cdot 10^{3}$ \\
\hline $4.0 \pi$ & 0.005 & 0.005 & 5.86 & 6.0 & $1.6 \cdot 10^{4}$ & $1.5 \cdot 10^{4}$ & $1.7 \cdot 10^{4}$ & $1.5 \cdot 10^{4}$ \\
\hline $10.0 \pi$ & 0.014 & 0.016 & 11.6 & 12.4 & $9.3 \cdot 10^{4}$ & $9.2 \cdot 10^{4}$ & $9.8 \cdot 10^{4}$ & $9.4 \cdot 10^{4}$ \\
\hline $50.0 \pi$ & 0.176 & 0.221 & 126.7 & 135.9 & $1.1 \cdot 10^{6}$ & $8.4 \cdot 10^{5}$ & $1.32 \cdot 10^{6}$ & $9.1 \cdot 10^{5}$ \\
\hline
\end{tabular}

even destabilize the closed-loop system. Results for the non-collocated input are depicted on Figures 22 and 23 , for $\theta_{1}^{d}(t)=6.0 \sin (0.75 \pi t)$. They show a big increase in both $e$ and the tracking error, compared with the collocated control: on Figure 19 we see that $\tilde{\theta}_{1}(t) \in[-1,1]$ degrees, while on Figure $22 \tilde{\theta}_{1}(t) \in[-12,6]$ degrees. In-between the peaks the tracking error for $\tau_{7}$ are also larger than with $\tau_{6}$.

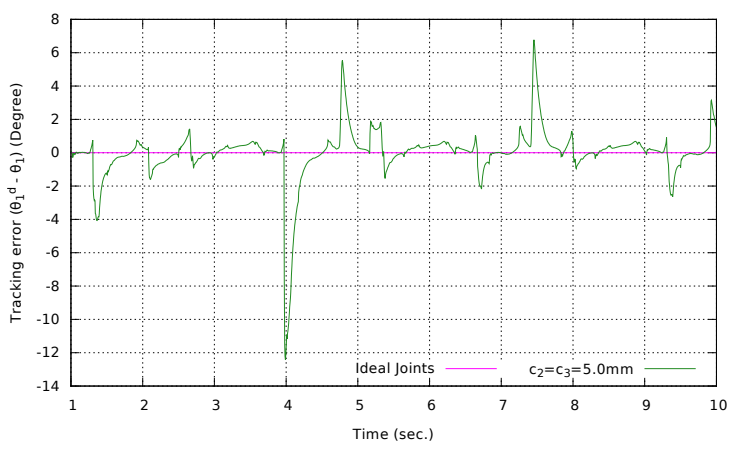

Figure 22: Crank-rocker with non-collocated passivity-based control $\tau_{7}$ : tracking error.

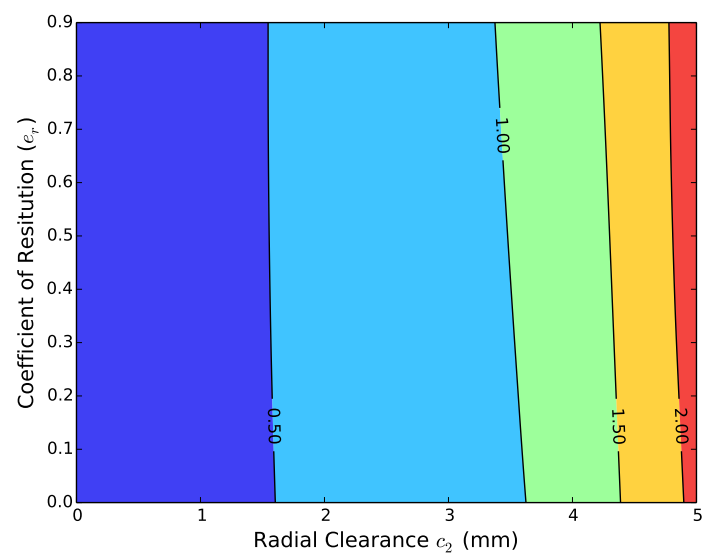

(a) .

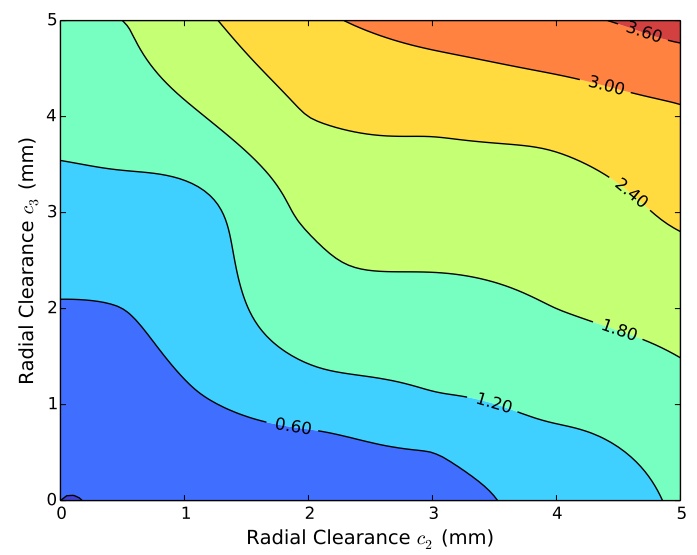

(b) .

Figure 23: Crank-rocker with non-collocated passivity-based control $\tau_{7}: e$. 


\subsection{Conclusions on sections 3, 4.1, 4.2 and 4.3}

Table 6 summarizes the tracking errors obtained with the above desired trajectories, for the torques $\tau_{3}, \tau_{4}, \tau_{5}$ and $\tau_{6}$, the three mechanisms and three cases (no play, one clearance and two clearances). In view of these data and the above results, the passivity-based controller $\tau_{6}$ is slightly better than the state linearization $\tau_{5}$. The two PD controllers, though they allow one to avoid the high sensitivity issues of the open-loop input $\tau_{2}$, yield too large tracking errors to possess practical interest in case precision is required (though the tracking error is drastically decreased using the velocity feedforward in $\tau_{4}$ ). Table 6 summarizes the results obtained for the maximum tracking errors with the four feedback controllers applied to the three mechanisms. Several comments arise, some of which just confirm previous ones: the compensation of smooth nonlinearities drastically improves the accuracy in all cases, for fixed control gains the PD controllers accuracy varies significantly depending on the system, while it does not for $\tau_{5}$ and $\tau_{6}$, for $\tau_{5}$ and $\tau_{6}$ the maximum tracking error doubles when a clearance at $J_{3}$ is added. We see also from Figures 10 (b), 13 (b), 15 (b) and 18 (b) that the performance decrease between the no play/play cases, is qualitatively the same for all collocated controllers in the presence of two clearances, while a small distortion occurs for the non-collocated input 23 (b). This shows that, at least for the chosen sinusoidal desired trajectories, a good predictability exists in such nonsmooth systems.

Table 6: Maximum tracking error on $[1,10] \mathrm{s}$ with feedback control, $K_{1}=2000, K_{2}=200, K=200$ and $\Lambda=10$.

\begin{tabular}{|c|c|c|c|c|}
\hline \multirow{3}{*}{ Four-bar mechanism } & \multirow{2}{*}{ Control torque } & \multicolumn{3}{|c|}{ Maximum tracking error (degrees) } \\
\cline { 3 - 5 } & & \multirow{2}{*}{ Ideal Joints } & \multicolumn{2}{|c|}{ Clearance in joints } \\
\cline { 3 - 5 } & & & $c_{2}=3.0 \mathrm{~mm}$ & $c_{2}=c_{3}=3.0 \mathrm{~mm}$ \\
\hline \multirow{3}{*}{ Crank-rocker } & $\tau_{3}$ & 82.5 & 84.2 & 85.2 \\
\cline { 2 - 5 } & $\tau_{4}$ & 2.98 & 5.68 & 6.68 \\
\cline { 2 - 5 } & $\tau_{5}$ & 0.003 & 0.7 & 1.2 \\
\cline { 2 - 5 } & $\tau_{6}$ & 0.003 & 0.66 & 1.12 \\
\hline \multirow{3}{*}{ Crank-crank } & $\tau_{3}$ & 103.3 & 105.22 & 106.92 \\
\cline { 2 - 5 } & $\tau_{4}$ & 25.4 & 27.32 & 29.02 \\
\cline { 2 - 5 } & $\tau_{5}$ & 0.004 & 0.73 & 1.31 \\
\cline { 2 - 5 } & $\tau_{6}$ & 0.004 & 0.68 & 1.22 \\
\hline \multirow{3}{*}{ Rocker-rocker } & $\tau_{3}$ & 34.57 & 36.07 & 37.07 \\
\cline { 2 - 5 } & $\tau_{4}$ & 1.79 & 3.29 & 1.26 \\
\cline { 2 - 5 } & $\tau_{5}$ & 0.003 & 0.67 & 1.19 \\
\cline { 2 - 5 } & $\tau_{6}$ & 0.003 & 0.61 & \\
\hline
\end{tabular}

\section{Conclusion}

A general methodology for modeling and simulation of multiple revolute joints with clearance in planar four-bar mechanisms has been presented and discussed in this work, and used to compare the robustness properties of several trajectory tracking feedback controllers (proportional-derivative, state linearization, and passivity-based control algorithms) with respect to such hard disturbances. The methodology is based on the nonsmooth dynamical approach, in which the interactions of the colliding bodies (journal and bearing) are modeled with unilateral constraints, restitution coefficients and Coulomb's friction. The combined projected Moreau-Jean event-capturing (time-stepping) scheme derived in [2] is 
used to solve numerically the contact-impact problem. It improves significantly the drift issue at the position level and allows to simulate persistent contact phases without spurious contact force and acceleration oscillations. It is noteworthy that the contact/impact models may be easily enhanced (taking into account static and dynamic friction, Stribeck effects, micro-displacements during sticking modes) while using the same dynamical and numerical framework. The major conclusions of this work is that collocated feedback improves drastically the system's dynamics (in the sense that trajectories of the clearance-free system and trajectories of the system with clearances, are close one to each other), and that the nonlinear controllers significantly improve the precision. Also the influence of the restitution (loss of kinetic energy at collisions) is negligible in our tested examples, while the clearances induce a symmetrical behaviour. The three-dimensional case should deserve attention, since it has considerable practical significance. In this setting cylindrical contact/impact models could be incorporated. Finally, the nonlinear feedback controllers which have been shown to be robust with respect to the hard disturbances represented by clearances, could be enhanced using ideas from [32]

\section{A Lagrangian formulation of four-bar mechanisms with reduced coordi- nates}

A four-bar mechanism is simplest form of closed-chain linkage and possesses one degree-of-freedom. The loop-closure constraints in the $x$ and $y$ coordinates are given as:

$$
\begin{aligned}
l_{4}+l_{3} \cos \theta_{3}-l_{2} \cos \theta_{2}-l_{1} \cos \theta_{1} & =0 \\
l_{3} \sin \theta_{3}-l_{2} \sin \theta_{2}-l_{1} \sin \theta_{1} & =0
\end{aligned}
$$

From (23) and (24) we can express $\theta_{2}$ and $\theta_{3}$ in terms of $\theta_{1}$. After some mathematical manipulations we get,

$$
c_{1}\left(\theta_{1}\right) \sin \left(\theta_{3}\right)+c_{2}\left(\theta_{1}\right) \cos \left(\theta_{3}\right)+c_{3}\left(\theta_{1}\right)=0
$$

where $c_{1}\left(\theta_{1}\right)=-2 l_{1} l_{3} \sin \theta_{1}, c_{2}\left(\theta_{1}\right)=-2 l_{3}\left(l_{4}-l_{1} \sin \theta_{1}\right), c_{3}\left(\theta_{1}\right)=l_{4}^{2}+l_{1}^{2}-l_{2}^{2}+l_{3}^{2}-2 l_{1} l_{4} \cos \theta_{1}$ Equation (25) can be solved in closed form as:

$$
p=\tan \frac{\theta_{3}}{2}, \quad \sin \theta_{3}=\frac{2 p}{1+p^{2}}, \quad \cos \theta_{3}=\frac{1-p^{2}}{1+p^{2}}
$$

From 25$)$ and 26 we have $\left(c_{3}-c_{2}\right) p^{2}+\left(2 c_{1}\right) p+\left(c_{2}+c_{3}\right)=0$, whose solution is given as $p=$ $\frac{-c_{1} \pm \sqrt{c_{1}^{2}+c_{2}^{2}-c_{3}^{2}}}{c_{3}-c_{2}}$. Then we obtain:

$$
\begin{aligned}
\theta_{3}\left(\theta_{1}\right) & =2 \arctan 2\left(-c_{1} \pm \sqrt{c_{1}^{2}+c_{2}^{2}-c_{3}^{2}}, c_{3}-c_{2}\right) \\
\theta_{2}\left(\theta_{1}, \theta_{3}\right) & =\arctan 2\left(-l_{1} \sin \theta_{1}+l_{3} \sin \theta_{3}, l_{3} \cos \theta_{3}-l_{1} \cos \theta_{1}\right)
\end{aligned}
$$


where the mapping $\arctan 2(\cdot, \cdot)$ is defined by

$$
\arctan 2(y, x)= \begin{cases}\arctan \frac{y}{x} & x>0 \\ \arctan \frac{y}{x}+\pi & y \geqslant 0, x<0 \\ \arctan \frac{y}{x}-\pi & y<0, x<0 \\ +\frac{\pi}{2} & y>0, x=0 \\ -\frac{\pi}{2} & y<0, x=0 \\ \text { undefined } & y=x=0\end{cases}
$$

Differentiating (23) and (24) with respect to time yields:

$$
\begin{array}{r}
l_{1} \sin \theta_{1} \dot{\theta}_{1}+l_{2} \sin \theta_{2} \dot{\theta}_{2}-l_{3} \sin \theta_{3} \dot{\theta}_{3}=0 \\
-l_{1} \cos \theta_{1} \dot{\theta}_{1}-l_{2} \cos \theta_{2} \dot{\theta}_{2}+l_{3} \cos \theta_{3} \dot{\theta}_{3}=0
\end{array}
$$

We can determine velocities $\dot{\theta}_{2}$ and $\dot{\theta}_{3}$ in terms of $\dot{\theta}_{1}$ as:

$$
\begin{aligned}
& \dot{\theta}_{2}=\frac{\partial \theta_{2}}{\partial \theta_{1}} \dot{\theta}_{1}=\frac{l_{1} \sin \left(\theta_{3}-\theta_{1}\right)}{l_{2} \sin \left(\theta_{2}-\theta_{3}\right)} \dot{\theta}_{1} \\
& \dot{\theta}_{3}=\frac{\partial \theta_{3}}{\partial \theta_{1}} \dot{\theta}_{1}=\frac{l_{1} \sin \left(\theta_{2}-\theta_{1}\right)}{l_{2} \sin \left(\theta_{2}-\theta_{3}\right)} \dot{\theta}_{1}
\end{aligned}
$$

The dynamical system is formulated from the Euler-Lagrange equations:

$$
\begin{gathered}
\frac{d}{d t}\left(\frac{\partial L\left(\theta_{1}, \dot{\theta}_{1}\right)}{\partial \dot{\theta}_{1}}\right)-\left(\frac{\partial L\left(\theta_{1}, \dot{\theta}_{1}\right)}{\partial \theta_{1}}\right)=\tau \\
L\left(\theta_{1}, \dot{\theta}_{1}\right)=T\left(\theta_{1}, \dot{\theta}_{1}\right)-V\left(\theta_{1}\right)
\end{gathered}
$$

where $L\left(\theta_{1}, \dot{\theta}_{1}\right) \in \mathbb{R}$ is the Lagrangian function, $T\left(\theta_{1}, \dot{\theta}_{1}\right)=\frac{1}{2} \dot{\theta}_{1}^{T} M\left(\theta_{1}\right) \dot{\theta}_{1}$ is the total kinetic energy, $V\left(\theta_{1}\right)$ is the total potential energy of the system and $\tau$ is the external torque. The Lagrangian function is given as:

$L\left(\theta_{1}, \theta_{2}, \theta_{3}, \dot{\theta}_{1}, \dot{\theta}_{2}, \dot{\theta}_{3}\right)=\left(T_{1}\left(\theta_{1}, \dot{\theta}_{1}\right)+T_{2}\left(\theta_{1}, \theta_{2}, \dot{\theta}_{2}, \dot{\theta}_{2}\right)+T_{3}\left(\theta_{3}, \dot{\theta}_{3}\right)\right)-\left(V_{1}\left(\theta_{1}\right)+V_{2}\left(\theta_{1}, \theta_{2}\right)+V_{3}\left(\theta_{3}\right)\right)$

where $T_{1}=0.25 m_{1} l_{1}^{2} \dot{\theta}_{1}^{2}+0.5 I_{1} \dot{\theta}_{1}^{2}, V_{1}=0.5 m_{1} l_{1} \mathbf{g} \sin \theta_{1}, V_{2}=m_{2} \mathbf{g}\left(l_{1} \sin \theta_{1}+0.5 l_{2} \sin \theta_{2}\right), V_{3}=$ $0.5 m_{3} l_{3} \mathbf{g} \sin \theta_{3}, T_{2}=0.5 m_{2}\left(l_{1}^{2} \dot{\theta}_{1}^{2}+0.5 l_{2}^{2} \dot{\theta}_{2}^{2}+l_{1} l_{2} \cos \left(\theta_{1}-\theta_{2}\right) \dot{\theta}_{1} \dot{\theta}_{2}\right)+0.5 I_{2} \dot{\theta}_{2}^{2}, T_{3}=0.25 m_{3} l_{3}^{2} \dot{\theta}_{3}^{2}+$ $0.5 I_{3} \dot{\theta}_{3}^{2}$, $\mathrm{g}$ is the gravitational acceleration. From 34 we infer the dynamics:

$$
M\left(\theta_{1}\right) \frac{d \dot{\theta}_{1}}{d t}+N\left(\theta_{1}, \dot{\theta}_{1}\right)+g\left(\theta_{1}\right)=\tau
$$

where:

$$
\begin{aligned}
& M\left(\theta_{1}\right)=2\left(J_{1}+J_{2} A_{1}^{2}+J_{3} A_{2}^{2}+0.5 m_{2} l_{1} l_{2} \cos \left(\theta_{1}-\theta_{2}\right)\right), g\left(\theta_{1}\right)=-\left(C_{1}+A_{1} C_{2}+A_{2} C_{3}\right) \\
& N\left(\theta_{1}, \dot{\theta}_{1}\right)=\left(2 J_{2} A_{1} A_{19}+2 J_{3} A_{2} A_{20}+A_{4}\left(A_{3} A_{19}+A_{1}\left(A_{11}+A_{1} A_{12}\right)\right)\right) \dot{\theta}_{1}^{2} \\
& A_{1}=\frac{l_{1} \sin \left(\theta_{3}-\theta_{1}\right)}{l_{2} \sin \left(\theta_{2}-\theta_{3}\right)}, A_{2}=\frac{l_{1} \sin \left(\theta_{2}-\theta_{1}\right)}{l_{2} \sin \left(\theta_{2}-\theta_{3}\right)}, A_{3}=\cos \left(\theta_{1}-\theta_{2}\right), A_{4}=0.5 m_{2} l_{1} l_{2}, \\
& A_{5}=\frac{\partial A_{1}}{\partial \theta_{1}}=\frac{-l_{1} \cos \left(\theta_{3}-\theta_{1}\right)}{l_{2} \sin \left(\theta_{2}-\theta_{3}\right)}, A_{6}=\frac{\partial A_{1}}{\partial \theta_{2}}=\frac{-l_{1} \sin \left(\theta_{3}-\theta_{1}\right) \cos \left(\theta_{2}-\theta_{3}\right)}{l_{2} \sin ^{2}\left(\theta_{2}-\theta_{3}\right)} \\
& A_{7}=\frac{\partial A_{1}}{\partial \theta_{3}}=\frac{-2 l_{1} \sin \left(\theta_{2}-\theta_{1}\right)}{-l_{2}+l_{2} \cos \left(2 \theta_{2}-2 \theta_{3}\right)}, A_{8}=\frac{\partial A_{2}}{\partial \theta_{1}}=\frac{-l_{1} \cos \left(\theta_{2}-\theta_{1}\right)}{l_{3} \sin \left(\theta_{2}-\theta_{3}\right)}
\end{aligned}
$$




$$
\begin{aligned}
& A_{9}=\frac{\partial A_{2}}{\partial \theta_{2}}=\frac{2 l_{1} \sin \left(\theta_{3}-\theta_{1}\right)}{-l_{3}+l_{3} \cos \left(2 \theta_{2}-2 \theta_{3}\right)}, A_{10}=\frac{\partial A_{2}}{\partial \theta_{3}}=\frac{l_{1} \sin \left(\theta_{2}-\theta_{1}\right) \cos \left(\theta_{2}-\theta_{3}\right)}{l_{3} \sin ^{2}\left(\theta_{2}-\theta_{3}\right)}, \\
& A_{11}=\frac{\partial A_{3}}{\partial \theta_{1}}=\sin \left(\theta_{2}-\theta_{1}\right), A_{12}=\frac{\partial A_{3}}{\partial \theta_{1}}=-\sin \left(\theta_{2}-\theta_{1}\right), A_{19}=A_{5}+A_{1} A_{6}+A_{2} A_{7}, A_{20}= \\
& A_{8}+A_{1} A_{9}+A_{2} A_{10}, J_{1}=0.5\left(0.33 m_{1} l_{1}^{2}+m_{2} l_{1}^{2}\right), J_{2}=0.17 m_{2} l_{2}^{2}, J_{3}=0.17 m_{3} l_{3}^{2}, C_{1}=-\left(0.5 m_{1} l_{1}+\right. \\
& \left.m_{2} l_{1}\right) \mathbf{g} \cos \theta_{1}, C_{2}=-0.5 m_{2} l_{2} \mathbf{g} \cos \theta_{2}, C_{3}=-0.5 m_{3} l_{3} \mathbf{g} \cos \theta_{3}
\end{aligned}
$$

\section{B Four-bar mechanism with clearance at joint $J_{2}$}

A four-bar mechanism with clearance in one revolute joint (see Figure 3(a)) possesses 3 degrees of freedom. The Lagrange dynamics in $(12)$ is given as follows:

$$
\begin{gathered}
M(q)=\left[\begin{array}{ccc}
\mathcal{J}_{1} & 0.5 N_{2} & 0 \\
0.5 N_{2} & \mathcal{J}_{2} & 0 \\
0 & 0 & \mathcal{J}_{3}
\end{array}\right], G_{1}(q)=\left[\begin{array}{ccc}
G_{11} & G_{12} & G_{13} \\
G_{21} & G_{22} & G_{23}
\end{array}\right], g(q)=\left[\begin{array}{c}
\left(0.5 m_{1}+m_{2}\right) F_{1} \\
0.5 m_{2} F_{2} \\
0.5 m_{3} F_{3}
\end{array}\right] \\
N(q, \dot{q})=\left[0.5 N_{1} \dot{\theta}_{2}^{2}, 0.5 N_{1} \dot{\theta}_{1}^{2}, 0\right]^{T}, B=[1,0,0]^{T}
\end{gathered}
$$

where:

$N_{2}=m_{2} l_{1} l_{2} \cos \left(\theta_{1}-\theta_{2}\right), F_{1}=\mathbf{g} l_{1} \cos \theta_{1}, F_{2}=\mathbf{g} l_{2} \cos \theta_{2}, F_{3}=\mathbf{g} l_{3} \cos \theta_{3}, \mathcal{J}_{1}=I_{1}+\left(0.25 m_{1}+\right.$ $\left.m_{2}\right) l_{1}^{2}, \mathcal{J}_{2}=I_{2}+0.25 m_{2} l_{2}^{2}, \mathcal{J}_{3}=I_{3}+\left(0.25 m_{3}\right) l_{3}^{2}, E=\sqrt{E_{x}^{2}+E_{y}^{2}}, E_{x}=-l_{4}-l_{3} \cos \theta_{3}+l_{2} \cos \theta_{1}+$ $l_{1} \cos \theta_{1}$,

$E_{y}=-l_{3} \sin \theta_{3}+l_{2} \sin \theta_{2}+l_{1} \sin \theta_{1}, G_{11}=\left(l_{1} \sin \theta_{1} E_{x}-l_{1} \cos \theta_{1} E_{y}\right) / E$,

$G_{21}=\left(\left(-l_{1} \sin \theta_{1} E_{y}-l_{1} \cos \theta_{1} E_{x}\right) / E\right)+r_{1}, G_{12}=\left(l_{2} \sin \theta_{2} E_{x}-l_{2} \cos \theta_{2} E_{y}\right) / E$,

$G_{13}=\left(-l_{3} \sin \theta_{3} E_{x}+l_{3} \cos \theta_{3} E_{y}\right) / E, G_{22}=\left(\left(-l_{2} \sin \theta_{2} E_{y}-l_{2} \cos \theta_{2} E_{x}\right) / E\right)-r_{2}$,

$G_{23}=\left(l_{3} \sin \theta_{3} E_{y}+l_{3} \cos \theta_{3} E_{x}\right) / E$

\section{Four-bar mechanism with clearances at joints $J_{2}$ and $J_{3}$}

A four-bar mechanism with clearance in two revolute joints (see Figure 3(b)) possesses 5 degrees of freedom. The unconstrained dynamics is that of three independent bodies and is given by:

$$
\begin{gathered}
M(q)=\left[\begin{array}{ccccc}
I_{1}+\left(0.25 m_{1}\right) l_{1}^{2} & 0 & 0 & 0 & 0 \\
0 & I_{2} & 0 & 0 & 0 \\
0 & 0 & I_{3}+\left(0.25 m_{3}\right) l_{3}^{2} & 0 & 0 \\
0 & 0 & 0 & m_{2} & 0 \\
0 & 0 & 0 & 0 & m_{2}
\end{array}\right], g(q)=\left[\begin{array}{c}
0.5 m_{1} \mathbf{g} l_{1} \cos \theta_{1} \\
0 \\
0.5 m_{3} \mathbf{g} l_{3} \cos \theta_{3} \\
0 \\
m_{2} \mathbf{g}
\end{array}\right], B=\left[\begin{array}{l}
1 \\
0 \\
0 \\
0 \\
0
\end{array}\right] \\
N(q, \dot{q})=\left[\begin{array}{l}
0 \\
0 \\
0 \\
0 \\
0
\end{array}\right], G_{1}(q)=\left[\begin{array}{lllll}
G_{11} & G_{12} & 0 & G_{14} & G_{15} \\
G_{21} & G_{22} & 0 & G_{24} & G_{25}
\end{array}\right], G_{2}(q)=\left[\begin{array}{lllll}
0 & \mathcal{G}_{12} & \mathcal{G}_{13} & \mathcal{G}_{14} & \mathcal{G}_{15} \\
0 & \mathcal{G}_{22} & \mathcal{G}_{23} & \mathcal{G}_{24} & \mathcal{G}_{25}
\end{array}\right]
\end{gathered}
$$

where: 


$$
\begin{aligned}
& G_{11}=\left(-X_{2} l_{1} \sin \theta_{1}+0.5 l_{1} l_{2} \sin \left(\theta_{1}-\theta_{2}\right)+Y_{3} l_{1} \cos \theta_{1}\right) C l_{1}, \\
& G_{21}=\left(\left(X_{2} l_{1} \cos \theta_{1}-0.5 l_{1} l_{2} \cos \left(\theta_{1}-\theta_{2}\right)+Y_{3} l_{1} \sin \theta_{1}-l_{1}^{2}\right) / C l_{1}\right)+r_{1} \\
& G_{12}=\left(-0.5 X_{2} l_{2} \sin \theta_{2}-0.5 l_{1} l_{2} \sin \left(\theta_{1}-\theta_{2}\right)+0.5 Y_{2} l_{2} \cos \theta_{2}\right) / V_{1} \text {, } \\
& G_{22}=\left(\left(0.5 X_{2} l_{2} \cos \theta_{2}-0.5 l_{1} l_{2} \cos \left(\theta_{1}-\theta_{2}\right)+0.5 Y_{2} l_{2} \sin \theta_{2}-0.25 l_{2}^{2}\right) / C l_{1}\right)-r_{2} \text {, } \\
& G_{14}=\left(-X_{2}+l_{1} \cos \theta_{1}+0.5 l_{2} \cos \theta_{2}\right) / C l_{1}, G_{15}=\left(-Y_{2}+l_{1} \sin \theta_{1}+0.5 l_{2} \sin \theta_{2}\right) / C l_{1} \text {, } \\
& G_{24}=\left(X_{2}-l_{1} \sin \theta_{1}-0.5 l_{2} \sin \theta_{2}\right) / C l_{1}, G_{25}=-\left(Y_{2}-l_{1} \cos \theta_{1}-0.5 l_{2} \cos \theta_{2}\right) / C l_{1} \text {, } \\
& \mathcal{G}_{12}=\left(-0.5 l_{4} l_{2} \sin \theta_{2}-0.5 l_{2} l_{3} \sin \left(\theta_{2}-\theta_{3}\right)+0.5 X_{2} l_{2} \sin \theta_{1}-0.5 Y_{2} l_{2} \cos \theta_{2}\right) / C l_{2} \text {, } \\
& \mathcal{G}_{13}=\left(l_{4} l_{3} \sin \theta_{3}+0.5 l_{2} l_{3} \sin \left(\theta_{2}-\theta_{3}\right)-X_{2} l_{3} \sin \theta_{3}+Y_{2} l_{3} \cos \theta_{3}\right) / C l_{2} \text {, } \\
& \mathcal{G}_{22}=\left(\left(0.5 l_{4} l_{2} \cos \theta_{2}-0.5 l_{2} l_{3} \sin \left(\theta_{2}-\theta_{3}\right)-0.5 X_{2} l_{2} \cos \theta_{1}+0.5 Y_{2} l_{2} \cos \theta_{2}-0.2 .5 l_{2}^{2}\right) / C l_{2}\right)+r_{3} \text {, } \\
& \mathcal{G}_{23}=\left(\left(-l_{4} l_{3} \cos \theta_{3}+0.5 l_{2} l_{3} \cos \left(\theta_{2}-\theta_{3}\right)+X_{2} l_{3} \cos \theta_{3}+Y_{2} l_{3} \sin \theta_{3}-l_{3}^{2}\right) / C l_{2}\right)-r_{4} \text {, } \\
& \mathcal{G}_{14}=\left(-X_{2}+l_{4}+l_{3} \cos \theta_{3}-0.5 l_{2} \cos \theta_{2}\right) / C l_{2}, \mathcal{G}_{24}=\left(Y_{2}-l_{3} \sin \theta_{3}+0.5 l_{2} \sin \theta_{2}\right) / C l_{2} \text {, } \\
& \mathcal{G}_{15}=\left(-Y_{2}+l_{3} \sin \theta_{3}-0.5 l_{2} \sin \theta_{2}\right) / C l_{2}, \mathcal{G}_{25}=\left(-X_{2}+l_{4}+l_{3} \cos \theta_{3}-0.5 l_{2} \cos \theta_{2}\right) / C l_{2} \text {, } \\
& C l_{1}=\sqrt{\left(X_{2}-0.5 l_{2} \cos \theta_{2}-l_{1} \cos \theta_{1}\right)^{2}+\left(Y_{2}-0.5 l_{2} \sin \theta_{2}-l_{1} \sin \theta_{1}\right)^{2}}, \\
& C l_{2}=\sqrt{\left(-l_{4}-l_{3} \cos \theta_{3}+0.5 l_{2} \cos \theta_{2}+X_{2}\right)^{2}+\left(-l_{3} \sin \theta_{3}+0.5 l_{2} \sin \theta_{2}+Y_{2}\right)^{2}}
\end{aligned}
$$

\section{Lyapunov functions}

The candidate Lyapunov function for the closed loop system in 21] is given as:

$$
\mathbf{V}(z)=\frac{1}{2}\left(\dot{\tilde{\theta}}_{1}^{2}+K_{1} \tilde{\theta}_{1}^{2}+C \tilde{\theta}_{1} \dot{\tilde{\theta}}_{1}\right)=\frac{1}{2} z^{T} P z
$$

where $P=\left[\begin{array}{cc}K_{1} & 0.5 C \\ 0.5 C & 1\end{array}\right]$, the position and velocity tracking errors are $\tilde{\theta}_{1} \triangleq\left(\theta_{1}-\theta_{1}^{d}\right)$ and $\dot{\tilde{\theta}}_{1} \triangleq\left(\dot{\theta}_{1}-\right.$ $\left.\dot{\theta}_{1}^{d}\right), z=\left(\tilde{\theta}_{1}, \dot{\tilde{\theta}}_{1}\right)^{T}$. Differentiating the Lyapunov function along the closed-loop system's trajectories gives:

$$
\begin{aligned}
\dot{\mathbf{V}}(z) & =\dot{\tilde{\theta}}_{1}\left(K_{2} \dot{\tilde{\theta}}_{1}-K_{1} \tilde{\theta}_{1}\right)+K_{1} \tilde{\theta}_{1} \dot{\tilde{\theta}}_{1}+C \dot{\tilde{\theta}}_{1}^{2}+C \tilde{\theta}_{1}\left(-K_{2} \dot{\tilde{\theta}}_{1}-K_{1} \tilde{\theta}_{1}\right) \\
& =k_{2} \dot{\tilde{\theta}}_{1}^{2}+C \dot{\tilde{\theta}_{1}^{2}}-C K_{2} \tilde{\theta}_{1} \dot{\tilde{\theta}}_{1}-C K_{1} \tilde{\theta}_{1}^{2}=-z^{T} Q z
\end{aligned}
$$

where $Q=\left[\begin{array}{cc}K_{2}-C & 0.5 C K_{2} \\ 0.5 C K_{2} & C K_{1}\end{array}\right]$. The matrices $Q$ and $P$ are positive definite if and only if the gains satisfy: $0<C<\frac{K_{1} K_{2}}{K_{1}+0.25 K_{2}}, K_{2}>C, K_{1}>\frac{C^{2}}{4}$. The closed-loop dynamics with the passivitybased controller in (22) admits the following Lyapunov-like function [8, p.404]:

$$
V(v)=\frac{1}{2} v^{T} M(q) v, \text { with } \dot{V}(v)=-v^{T} K v
$$

It allows to prove (in the ideal no-clearance case) that all trajectories are bounded and the tracking errors globally asymptotically converge to zero.

\section{References}

[1] M. Abadie. Dynamic simulation of rigid bodies: Modelling of frictional contact. In B. Brogliato, editor, Impacts in Mechanical Systems: Analysis and Modelling, volume 551 of Lecture Notes in Physics (LNP), pages 61-144. Springer, 2000.

[2] V. Acary. Projected event-capturing time-stepping schemes for nonsmooth mechanical systems with unilateral contact and Coulomb's friction. Computer Methods in Applied Mechanics and Engineering, 256:224-250, 2013. 
[3] V. Acary and B. Brogliato. Numerical Methods for Nonsmooth Dynamical Systems. Applications in Mechanics and Electronics. Lecture Notes in Applied and Computational Mechanics 35. Berlin: Springer. xxi, 525 p. , 2008.

[4] D. Angeli. Input-to-state stability of PD-controlled robotic systems. Automatica, 35:1285-1290, 1999.

[5] P. Ballard. The dynamics of discrete mechanical systems with perfect unilateral constraints. Archive for Rational Mechanics and Analysis, 154:199-274, September 2000.

[6] P. Ballard and S. Basseville. Existence and uniqueness for frictional unilateral contact with Coulomb friction: a model problem. ESAIM: M2AN Mathematical Modelling and Numerical Analysis, 39:59-75, September 2005.

[7] B. Brogliato. Nonsmooth Mechanics: Models, Dynamics and Control. Springer-Verlag, London, 2nd edition, 1999.

[8] B. Brogliato, R. Lozano, B. Maschke, and O. Egeland. Dissipative Systems Analysis and Control: Theory and Applications. Springer, 2nd edition, 2007.

[9] Q. Chen, H. Chen, Y. Wang, and P.Y. Woo. Global stability analysis of some trajectory-tracking control schemes of robotic manipulators. Journal of Robotic Systems, 18(2):69-75, 2001.

[10] M.O.T. Cole, T. Wongratanaphisan, R. Pongvuthithum, and W. Fakkaew. Controller design for flexible structure vibration suppression with robustness to contacts. Automatica, 44:2876-2883, 2008.

[11] M. di Bernardo, C.J. Budd, A.R. Champneys, and P. Kowalczyk. Piecewise Smooth Dynamical Systems: Theory and Applications, volume 163 of Applied Mathematical Sciences. Springer Verlag, London, 2008.

[12] F.B. Duarte and J. Tenreiro Machado. Describing function of two masses with backlash. Nonlinear Dynamics, 56:409413, 2009.

[13] S. Dubowsky and F. Freudenstein. Dynamic analysis of mechanical systems with clearances-part 1: Formation of dynamic model. Journal of Engineering for Industry, 93:305-309, 1971.

[14] S. Dubowsky and F. Freudenstein. Dynamic analysis of mechanical systems with clearances-part 2: Dynamic response. Journal of Engineering for Industry, 93:310-316, 1971.

[15] R. Dzonou and M.D.P. Monteiro Marques. A sweeping process approach to inelastic contact problems with general inertia operators. European Journal of Mechanics A/Solids, 26(3):474-490, 2007.

[16] R. Dzonou, M.D.P. Monteiro Marques, and L. Paoli. A convergence result for a vibro-impact problem with a general inertia operator. Nonlinear Dynamics, 58(1-2):361-384, 2009.

[17] S.W.E. Earles and C.L.S. Wu. Motion analysis of a rigid link mechanism with clearance at a bearing using Lagrangian mechanics and digital computation. Mechanisms (Proceedings, Institution of Mechanical Engineers), London, 22:83-89, 1973.

[18] S. Erkaya and I. Uzmay. Investigation on effect of joint clearance on dynamics of four-bar mechanism. Nonlinear Dynamics, 58:179-198, 2009.

[19] F. Farahanchi and S.W. Shaw. Chaotic and periodic dynamics of a slider-crank mechanism with slider clearance. Journal of sound and vibration, 177(3):307-324, 1994.

[20] P. Flores. A parametric study on the dynamic response of planar multibody systems with multiple clearance joints. Nonlinear Dynamics, 61(4):633-653, 2010.

[21] P. Flores and J. Ambrósio. Revolute joints with clearance in multibody systems. Computers \& Structures, 82(17):13591369, 2004.

[22] P. Flores, J. Ambrosio, J.C. Pimenta Claro, and H.M. Lankarani. Kinematics and Dynamics of Multibody Systems with Imperfect Joints, volume 34 of Lecture Notes in Applied and Computational Mechanics. Springer Verlag, Heidelberg, 2008.

[23] P. Flores, C.S. Koshy, H.M. Lankarani, J. Ambrosio, and J.C.P. Claro. Numerical and experimental investigation on multibody systems with revolute clearance joints. Nonlinear Dynamics, 65:383-398, 2011.

[24] P. Flores, R. Leine, and C. Glocker. Modeling and analysis of planar rigid multibody systems with translational clearance joints based on the non-smooth dynamics approach. Multibody System Dynamics, 23:165-190, 2010.

[25] H. Funabashi, K. Ogawa, and M. Horie. A dynamic analysis of mechanisms with clearances. Bulletin of JSME, 21(161):1652-1659, 1978.

[26] C. Glocker. Set-Valued Force Laws, volume 1 of Lecture Notes in Applied Mechanics. Springer Verlag, Berlin Heidelberg, 2001.

[27] A. Gummer and B. Sauer. Modeling planar slider-crank mechanisms with clearance joints in RecurDyn. Multibody System Dynamics, 31:127-145, 2014. 
[28] R.S. Haines. Survey: 2-dimensional motion and impact at revolute joints. Mechanism and Machine Theory, 15(5):361$370,1980$.

[29] M. Horie, H. Funabashi, K. Ogawa, and H. Abe. Dynamic characteristics of planar link mechanisms with clearances: Conditions of separation occurrence between pairing elements. Bulletin of JSME, 29(252):1888-1894, 1986.

[30] M. Jean. The non-smooth contact dynamics method. Computer Methods in Applied Mechanics and Engineering, 177(3):235-257, 1999.

[31] M. Jean and J.J. Moreau. Dynamics in the presence of unilateral contacts and dry friction: a numerical approach. In G. del Piero and F. Maceri, editors, Unilateral Problems in Structural Analysis II, number 304 in CISM Courses and Lectures, pages 151-196. Springer Verlag, 1987.

[32] M. Mata Jimenez and B. Brogliato. Analysis of PD and nonlinear control of mechanical systems with dynamic backlash. Journal of Vibration and Control, 9(1):119-156, 2003.

[33] R. Kelly and R. Carelli. A class of nonlinear PD-type controllers for robot manipulators. Journal of Robotic Systems, 13(12):793-802, 1996.

[34] R. Kikuuwe. Alternative proofs of four stability properties of rigid-link manipulators under PID position control. Robotica, 31(1):113-122, 2012.

[35] C.S. Koshy, P. Flores, and H.M. Lankarani. Study of the effect of contact force model on the dynamic response of mechaincal systems with dry clearance joints: computational and experimental approaches. Nonlinear Dynamics, 73:325338, 2013.

[36] A. Krinner and T. Thümmel. Non-smooth behaviour of a linkage mechanism with revolute clearance joints. In New Advances in Mechanisms, Transmissions and Applications, pages 233-241. Springer, 2014.

[37] A. Lagerberg. A literature survey on control of automotive powertrains with backlash. Control and Automation Laboratory, Chalmers University of Technology, Göteborg, Sweden, R013/2001.

[38] A. Lagerberg and B. Egardt. Backlash estimation with application to automotive powertrains. IEEE Transactions on Control Systems Technology, 15(3):483-493, 2007.

[39] R.I. Leine and H. Nijmeijer. Dynamics and Bifurcations of Non-Smooth Mechanical Systems, volume 18 of Lecture Notes in Applied and Computational Mechanics. Springer Verlag, Berlin Heidelberg, 2004.

[40] X. Li, X. Ding, and G.S. Chirikjian. Analysis of angular-error uncertainty in planar multiple-loop structures with joint clearances. Mechanism and Machine Theory, 91:69-85, 2015.

[41] C. Liu, Q. Tian, and H. Hu. Dynamics and control of a spatial rigid-flexible multibody system with multiple cylindrical clearance joints. Mechanism and Machine Theory, 52:106-129, 2012.

[42] L. Menini and A. Tornambé. State estimation of (otehrwise unobservable) linear mechanical systems through the use of non-smooth impacts: the case of two mating gears. Automatica, 38:1823-1826, 2002.

[43] J.J. Moreau. Standard inelastic shocks and the dynamics of unilateral constraints. In CISM Courses and Lectures, volume 288, pages 173-221. Springer Verlag, 1985. Preprint 84-2, February 1984, Laboratoire de Mécanique Générale des Milieux Continus, université des Sciences et Techniques du Languedoc, France.

[44] J.J. Moreau. Unilateral contact and dry friction in finite freedom dynamics. In J.J. Moreau and P.D. Panagiotopoulos, editors, Nonsmooth Mechanics and Applications, pages 1-82. CISM 302, Spinger Verlag, 1988.

[45] J.J. Moreau. Some numerical methods in multibody dynamics: Application to granular materials. Eur. J. Mech./ A Solids, supp.(13):93-114, 1994.

[46] J.J. Moreau. Numerical aspects of the sweeping process. Computer Methods in Applied Mechanics and Engineering, 177:329-349, 1999.

[47] A. Müller. Internal preload control of redundantly actuated parallel manipulators -Its application to backlash avoiding control. IEEE Transactions on Robotics, 21(4):668-677, 2005.

[48] M. Nordin, J. Galic, and P.O. Gutman. New models for backlash and gear play. International Journal of Adaptive Control and Signal Processing, 1:9-63, 1997.

[49] M. Nordin and P.O. Gutman. Controlling mechanical systems with backlash-a survey. Automatica, 38:1633-1649, 2002.

[50] A.A. Olyaei and M.R. Ghazavi. Stabilizing slider-crank mechanism with clearance joints. Mechanism and Machine Theory, 53:17-29, 2012.

[51] A.A. Olyaei and M.Z. Ghazavi. Stabilizing slider-crank mechanism with clearance joints. Mechanism and Machine Theory, 53:17-29, 2012.

[52] J.C. Garcia Orden. Analysis of joint clearances in multibody systems. Multibody System Dynamics, 13:401-420, 2005. 
[53] L. Paoli. A proximal-like method for a class of second order measure-differential inclusions describing vibro-impact problems. Journal of Differential Equations, 250(1):476 - 514, 2011.

[54] E. Pennestri, P.P. Valentini, and L. Vita. Multibody dynamics simulation of planar linkages with Dahl friction. Multibody System Dynamics, 17:321-347, 2007.

[55] C. Pereira, A. Ramalho, and J. Ambrosio. An enhanced cylindrical contact force model. Multibody System Dynamics, 2015. DOI: 10.1007/s11044-015-9463-x.

[56] F. Pfeiffer and C. Glocker. Multibody Dynamics with Unilateral Contacts. Non-linear Dynamics. John Wiley \& Sons, 1996.

[57] S. Rahmanian and M.R. Ghazavi. Bifurcation in planar slider-crank mechanism with revolute clearance joint. Mechanism and Machine Theory, 91:86-101, 2015.

[58] P. Ravn. A continuous analysis method for planar multibody systems with joint clearance. Multibody System Dynamics, 2(1):1-24, 1998.

[59] J. Rhee and A. Akay. Dynamic response of a revolute joint with clearance. Mechanism and Machine Theory, 31(1):121134, 1996.

[60] V. Santibanez and R. Kelly. PD control with feedforward compensation for robot manipulators: analysis and experimentation. Robotica, 19(1):11-19, 2001.

[61] L.D. Seneviratne and S.W.E. Earles. Chaotic behaviour exhibited during contact loss in a clearance joint of a four-bar mechanism. Mechanism and Machine Theory, 27(3):307-321, 1992.

[62] D.E. Stewart. Convergence of a time-stepping scheme for rigid-body dynamics and resolution of painlevé's problem. Archives of Rational Mechanics Anal., 145(3):215-260, 1998.

[63] C. Studer. Numerics of Unilateral Contacts and Friction. - Modeling and Numerical Time Integration in Non-Smooth Dynamics, volume 47 of Lecture Notes in Applied and Computational Mechanics. Springer Verlag, 2009.

[64] T. Thümmel and K. Funk. Multibody modelling of linkage mechanisms including friction, clearance and impact. In Proceedings of the 10th World Congress on the Theory of Machines and Mechanisms in Oulu, June 20 to 24, volume 4, pages 1387-1392. Oulu University Press, Finland, 1999.

[65] Y. Tang, Z. Chang, X. Dong, Y. Hu, and Z. Yu. Nonlinear dynamics and analysis of a four-bar linkage with clearance. Front. Mech. Eng., 8(2):160-168, 2013.

[66] G. Tao and P.V. Kokotovic. Adaptive control of systems with backlash. Automatica, 29(2):323-335, 1993.

[67] G. Tao and P.V. Kokotovic. Adaptive control of plants with unknown hystereses. IEEE Transactions on Automatic Control, 40(2):200-212, 1995.

[68] T. Thümmel. Experimentelle Mechanismendynamik: Messung, Modellierung, Simulation, Verifikation, Interpretation und Beeinflussung typischer Schwingungsphänomene an einem Mechanismenprüfstand. PhD thesis, München, Technische Universität München, Habil.-Schr., 2012, 2012.

[69] T. Thümmel and L. Ginzinger. Measurements and simulations of a crank and rocker mechanism including friction, clearance and impacts. In In Proceedings of the IX. International Conference on the Theory of Machines and Mechanisms in Liberec/Czech Republic, Aug.31-Sept.2004, pages 763-768. Technical University of Liberec, Department of Textile Machine Design, 2004.

[70] T. Thümmel and M. Roßner. Introduction to modelling and parameter identification methodology of linkages by measurements and simulation. In Proceedings of the 13th World Congress in Mechanism and Machine Science, Guanajuato, Mexico, 19-25 June, volume IMD-123, 2011.

[71] T. Thümmel, J. Rutzmoser, and H.Ulbrich M. Robner. Friction modeling and parameter value estimation of mechanisms. In The 2nd Joint International Conference on Multibody Systems Dynamics, May 29-June 1,2012, Stuttgart, Germany, pages 302-312. University of Stuttgart, Institute of Engineering and Computational Mechanics, 2012.

[72] K.L. Ting, J. Zhu, and D. Watkins. The effects of joint clearance on position and orientation deviation of linkages and manipulators. Mechanism and Machine Theory, 35(3):391-401, 2000.

[73] S.M. Varedi, H.M. Daniali, and M. Dardel. Dynamic synthesis of a planar slider-crank mechanism with clearances. Nonlinear Dynamics, 2014. DOI: 10.1007/s11071-014-1762-x.

[74] S. Yan, W. Xiang, and L. Zhang. A comprehensive model for 3D revolute joints with clearances in mechanical systems. Nonlinear Dynamics, 2014. DOI: 10.1007/s11071-014-1870-7.

[75] H. Zabiri and Y. Samyudia. A hybrid formulation and design of model predictive control for systems under saturation and backlash. Journal of Process Control, 16:693-709, 2006. 
[76] H. Zhang, B. Brogliato, and C. Liu. Dynamics of planar rocking-blocks with Coulomb friction and unilateral constraints: comparisons between experimental and numerical data. Multibody System Dynamics, 32(1):1-25, 2014. 